\title{
Cross-linking of double oil-in-water-in-oil emulsions: a new way for fragrance encapsulation with tunable sustained release.
}

\author{
Margot Stasse ${ }^{\mathrm{a}, \mathrm{b}, \mathrm{c}}$, Eric Laurichesse ${ }^{\mathrm{b}}$, Magali Vandroux ${ }^{\mathrm{c}}$, Tiphaine Ribaut ${ }^{\mathrm{c}}$, Valérie \\ Héroguez $^{\mathrm{a}^{*}}$, Véronique Schmitt ${ }^{\mathrm{b}^{*}}$
}

aaboratoire de chimie des polymères organiques Univ. Bordeaux, CNRS, Bordeaux INP, UMR 5629, 16 avenue Pey-Berland, 3600 Pessac, France

${ }^{\mathrm{b}}$ Centre de recherche Paul Pascal, 115 avenue du Dr Albert Schweitzer 33600 Pessac France

${ }^{\mathrm{c}}$ Takasago Europe Perfumery Laboratory 12 rue Torricelli 75017 Paris France

* corresponding authors

e-mail addresses: $\quad$ valerie.heroguez@enscbp.fr (Valérie Héroguez)

veronique.schmitt@crpp.cnrs.fr (Véronique Schmitt)

\section{Abstract}

This paper describes a new route to synthesize polymeric capsules with interesting and diverse diffusive and mechanical properties. Such capsules were obtained by free radical cross-linking copolymerization of the intermediate water phase of a double oil-in-water-in-oil (O/W/O) emulsion. Such a strategy allows separating the locus of polymerization from the fragrance- containing compartment. Indeed fragrance molecules are known to be reactive. The encapsulated species, also corresponding to the innermost oil, was a model fragrance composed of more than 10 various molecules usually used in perfumery. In a first step, the double emulsion containing a hydrophilic monomer, either Methacrylamide or Oligo(ethyleneglycol) methacrylate, and a hydrophilic cross-linker, either Tetra(ethyleneglycol) diacrylate or N,N-Methylene-bis-Acrylamide, was formulated. The influence of the cross-linker agent on the feasibility of the double emulsion was examined. Then, in a second part, the polymerization was performed. Four different systems of capsules were developed and compared in terms of diffusive and mechanical properties. We show that changing the monomer /cross-linker couple allowed modulating the capsule properties. 
Keywords: microcapsule, double emulsion, free radical polymerisation, fragrance, release, encapsulation

Highlights:

- Matrix microcapsules elaboration from double fragrance-in-aqueous phase-in perfumery solvent emulsion

- Polymerization and cross-linking of the intermediate aqueous phase

- Modularity of fragrance release from microcapsules thanks to the choice of the crosslinker

- Model fragrance composed of a blend of 13 molecules current in perfumery

Graphical abstract

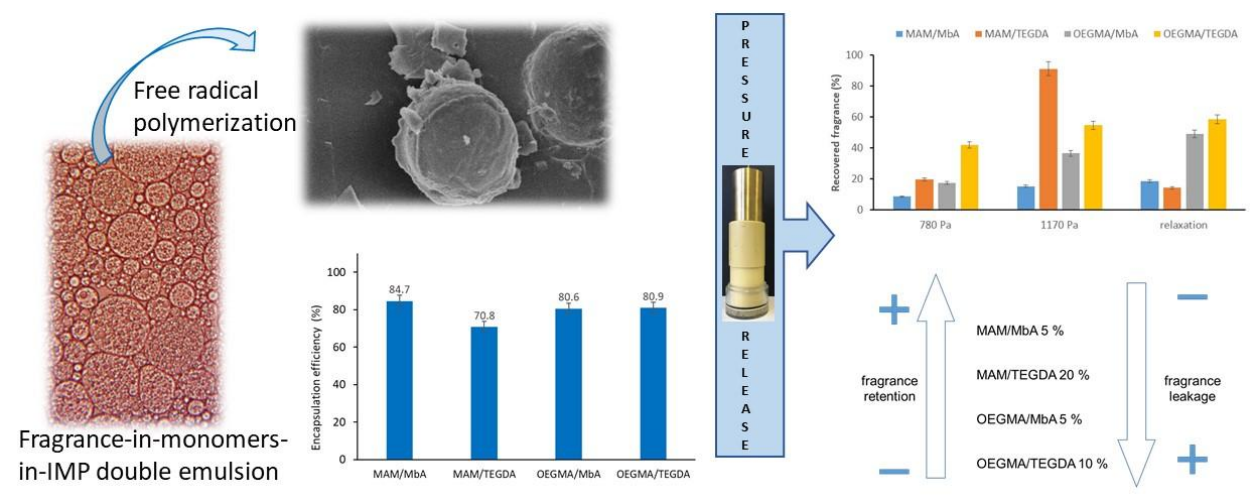

Abbreviations:

HS: hydrophilic stabilizer

LS: lipophilic stabilizer

PGPR: polyglycerol polyricinoleate

IPM: isopropyl myristate

HLB: Hydrophilic — Lipophilic Balance

wt $\%$ : weight $\%$

OEGMA: Oligo(ethyleneglycol) methacrylate

MAM: Methacrylamide

TEGDA: Tetra(ethyleneglycol) diacrylate

MbA: N,N-Methylene-bis-Acrylamide 


\section{Introduction}

Since the first applications in the 50s for carbonless copy paper [1], microcapsules have been introduced in many industries. In agriculture, pesticides are encapsulated to be released with time [2-4] allowing them to be more effective and safer to both operators and environment [5,6]. In food, flavors are encapsulated for taste masking, for protecting ingredients from the external environment or oxidation [7-9] or as additional vitamin provider [10,11]. In stickers, cardboard, books and magazines, scratch and sniff technology is used to provide a sensory experience to the reader. In textiles, active-containing microcapsules are grafted on textile fibers to release their content during the wearing of clothing [12,13]. In pharmaceutical applications [14,15], microcapsules can also offer various advantages including: an easy administration of the drug, a pre-programmed release profile to meet the therapeutic needs of the patient and an effective protection of drug against (e.g. enzymatic) degradation. Recent silica porous beads have been used for cascade enzymatic reactions [16]. For building applications, microcapsules containing phase change materials $[17,18]$ are also developed. The encapsulation of highly viscous bitumen recently led to powdered bitumen exhibiting the advantages of being flowable at room temperature, easily transportable, storable...[19,20]

First fragrance encapsulation techniques have been developed [21] in the 70s with the aim to control the duration of fragrance release and to protect the fragrance from the environment. Home and personal care applications, in which they are intended to be used, are very restrictive, and few microcapsules types can satisfy the required properties. The most widespread capsules are matrix starch capsules [22] manufactured by spray-drying processes and core-shell capsules synthesized thanks to in-situ polymerization (melamine-formaldehyde [23-25], urea-formaldehyde microcapsules [26] or microcapsules synthesized through freeradical suspension polymerization [27-30]), interfacial polycondensation (polyurethane microcapsules) or coacervation [31]. 
All these capsules exhibit advantages and disadvantages and cannot be used in all applications. Starch capsules disintegrate in contact with water and are thus dedicated to specific applications such as water-free deodorants or cat liters. Other capsules are frictionsensitive capsules. Capsules synthesized by coacervation have generally the advantage to be based on natural and biodegradable polymers but are not sufficiently cross-linked to enough retain the fragrance when used in surfactant-rich liquid products; they can thus only be used in deodorants. Melamine- or urea-formaldehyde capsules contain residual formaldehyde, which is a carcinogenic molecule and cannot be considered for cosmetic applications. Microcapsules synthesized by interfacial polymerization or in-situ polymerization exhibit the drawback that a part of the reactants is located at the interface or in the same phase than the encapsulated species and some of the fragrance molecules functions can interact with the encapsulation process and/or be degraded leading to an alteration of olfactory notes.

We propose herein a new encapsulation route based on the formulation of double emulsions [32,33] and more precisely fragrance-in-water-in-perfumery solvent that allows the compartmentalization between the polymerization locus and the fragrance and we vary the proportion and type of cross-linker. Capsules were synthesized by free radical polymerization of the intermediate water phase of a double fragrance-in-water-in-oil emulsion containing monomers, cross-linkers and initiator. The direct fragrance-in-water emulsion was stabilized with Tergitol 15-S-12 a hydrophilic surfactant (HS) and the inverted water-in-oil emulsion by Polyglycerol polyricinoleate (PGPR) a lipophilic surfactant (LS) [32]. Two monomers were chosen: Oligo(ethyleneglycol) methacrylate (OEGMA) and the Methacrylamide (MAM) [33]. These monomers were selected owing their hydrophilicity, their ability to polymerize only in water and their biocompatibility. However, the obtained capsules exhibited limitations [33]. Indeed, the presence of only monofunctionnal monomers led to the synthesis of a matrix with a low density, that could be responsible for fragrance leakage [33]. To improve the barrier 
effect and control the release of the fragrance, we suggest in this work to crosslink the polymerized intermediate phase in order to obtain a 3D network. It becomes thus possible to modulate the diffusive properties of the capsules by simply changing the monomer/crosslinker couple or the cross-linker to monomer ratio. Free-radical copolymerization was preferred because of its easy transposition on an industrial scale. Two different crosslinkers (difunctional monomers) were considered: Tetra(ethyleneglycol) diacrylate (TEGDA) and the N,N-Methylene-bis-Acrylamide (MbA). The 4 units of ethylene glycol (TEGDA) between the two acrylate functions is expected to confer flexibility to the $3 \mathrm{D}$ network whereas the $\mathrm{MbA}$ can add physical cross-linking [33] due to the possible hydrogen bonding [34].

First, the formulation of the double emulsion in presence of both the monomers and the crosslinkers at different concentrations was studied in order to determine the best compositions for the double emulsions. Then, for the selected compositions, the free radical polymerizations were proceeded to obtain the microcapsules. Finally, the intrinsic properties of the synthesized capsules were examined: fragrance encapsulation efficiency, fragrance release across the capsules in closed and open vials and mechanical properties that are all representative of end-use properties.

\section{Materials and Methods}

\section{II.1. Materials}

All the reagents were purchased and used without further purification, Polyglycerol Polyricinoleate (PGPR) with a molar mass of 3000 g.mol ${ }^{-1}$ was provided by Paalsgard. Tergitol 15-S-12 was purchased from Sigma-Aldrich (molar mass $=760$ g.mol ${ }^{-1}$ and HLB $=$ 14.5). The outer perfumery oil was Isopropyl Myristaste (IPM, density equal to $0.850 \mathrm{~g} \cdot \mathrm{mL}^{-1}$ ) provided by Takasago European Perfumery Laboratory (T.E.P.L). The innermost oil was a fragrance specially composed for the study and representative of usually used fragrances. It is composed of 13 molecules (Supporting Information 1) with alkene, aldehyde, hydroxyl, 
ketone, ester moeties. Its properties are summarized in Table1. Oligo(ethyleneglycol) methacrylate $\left(\mathrm{M}_{\mathrm{n}}=360 \mathrm{~g} \cdot \mathrm{mol}^{-1}\right.$ contains $500-800 \mathrm{ppm}$ of monomethyl ether hydroquinone $)$, Methacrylamide (Molar mass $=85.1$ g.mol ${ }^{-1}, 98 \%$ ), Tetraethyleneglycol diacrylate (molar mass $\left.=302.3 \mathrm{~g} \cdot \mathrm{mol}^{-1}, \geq 90 \%\right), \mathrm{N}, \mathrm{N}$, Methylene-bis-Acrylamide (Molar mass $=154.2 \mathrm{~g} \cdot \mathrm{mol}^{-1}$, 99\%) Sodium Chloride $(\mathrm{NaCl})$, Potassium Persulfate (KPS) have been purchased from Sigma-Aldrich. Milli-Q water was used in all emulsion preparation.

Table 1: Properties of the fragrance [32,33]

\begin{tabular}{|c|c|c|c|}
\hline $\log P^{(a)}$ & Refractive index $^{(\mathrm{b})}$ & Density $^{(c)}$ & $\begin{array}{l}\text { Interfacial tension versus } \\
\text { water }(\mathrm{mN} / \mathrm{m})^{(\mathrm{d})}\end{array}$ \\
\hline 3.52 & 1.475 & 0.887 & 34.6 \\
\hline
\end{tabular}

(a) $\log \mathrm{P}$ is defined by $\log \mathrm{P}=\log \left(\mathrm{C}_{\text {octanol }} / \mathrm{C}_{\text {water }}\right)$, $\mathrm{P}$ denotes the partition coefficient between octanol and water, $\mathrm{C}_{\text {octanol }}$ and $\mathrm{C}_{\mathrm{water}}$ are the concentrations of the species in octanol and water respectively. As the fragrance is a mixture of 13 molecules, a mean $\operatorname{LogP}$ is calculated as the molar average $\log \mathrm{P}$ [35]. For the model fragrance considered here, the average value of $\log \mathrm{P}$ as well $\log \mathrm{P}$ of each molecule are positive meaning that the fragrance and its compounds are more soluble in octanol than in water.

(b) measured with an Abbé refractometer

${ }^{\text {(c) }}$ measured with a densimeter

${ }^{\text {(d) }}$ measured with the weighted method

II. 2 Formulation of the double oil-in-water-in-oil emulsion

Double oil-in-water-in-oil emulsions were prepared following a two-step procedure as described in a previous work [32]

\section{II.2.1. Direct emulsion}

First a polydisperse fragrance-in-water emulsion was obtained by manually incorporating the fragrance $(90 \mathrm{wt} \%)$ into an aqueous phase composed of salted water 
$(\mathrm{NaCl})$ and Tergitol $15-\mathrm{s}-12$ at $30 \mathrm{wt} \%$ with respect to the water phase. In other words, for $100 \mathrm{~g}$ of a direct emulsion, the composition was the following: $90 \mathrm{~g}$ of fragrance dispersed in a water phase composed of $0.04 \mathrm{~g}$ of $\mathrm{NaCl}, 3 \mathrm{~g}$ of Tergitol 15-s-12 and $6.96 \mathrm{~g}$ of $\mathrm{H}_{2} \mathrm{O}$. This crude premixed emulsion was then sheared by means of a Couette cell (produced by AdemTech) to reduce the drops size distribution width [36]. The Couette cell is composed of two concentric cylinders. The inner cylinder of radius $\mathrm{Ri}=$ $20 \mathrm{~mm}$ rotated with a speed that we fixed at $680 \mathrm{rpm}$. The gap with the outer static cylinder was $100 \mu \mathrm{m}$ so that the shear rate was $14280 \mathrm{~s}^{-1}$. The emulsion was injected in the device by a syringe pump so that the shearing time of the emulsion was of the order of $10 \mathrm{~s}$. This delay was sufficient for the Rayleigh instability to develop leading to emulsions with narrow drop size distributions [36]. We chose to concentrate the direct emulsion up to $90 \mathrm{wt} \%$ in order to optimize the fragmentation into small droplets. Indeed, the Rayleigh instability is especially efficient at such high fractions [36]. The resulting direct emulsion was characterized by a volume average diameter of $1.0 \mu \mathrm{m}$ with a narrow size distribution $\left(\mathrm{P}_{\mathrm{d}}=27 \%\right)$ as it can be seen on Figure S2 (See supporting informations 3 for the definition of the volume average diameter and the polydispersity index $\mathrm{P}_{\mathrm{d}}$ ). The fragmentation was efficient as, in average, one drop of the premixed emulsion fragmented into 86 droplets.

\section{II.2.2. Double emulsion}

In a second step, the just described direct emulsion was diluted by a factor 4.5 with an aqueous phase composed of HS, initiator, monomers and possibly cross-linker, to reach $20 \mathrm{wt} \%$ of fragrance droplets. This dilution step allowed tuning the intermediate aqueous phase composition (nature and amount of monomer and cross- 
linker) keeping the first emulsification step unmodified. This dilute emulsion was manually incorporated in IPM oil containing $10 \mathrm{wt} \%$ of PGPR (with respect to the oil outer phase) to reach a fraction of $75 \mathrm{wt} \%$. As a consequence, the fragrance droplets represented $\phi_{\mathrm{d}}=20 \mathrm{wt} \%$ of the aqueous globules, themselves corresponding to $\phi_{\mathrm{G}}=75 \mathrm{wt} \%$ of the total sample. This formulation therefore allowed an encapsulation of $15 \mathrm{~g}$ of fragrance/100 $\mathrm{g}$ of the total sample. A previous formulation work [32] showed that the fragrance could be incorporated up to $50 \mathrm{wt} \%$ in the globules therefore corresponding to an encapsulation load of $37.5 \mathrm{wt} \%$ of the total sample. Nevertheless in the present paper the fragrance load with respect to total sample was fixed at $15 \mathrm{wt} \%$ whatever the monomers and cross-linkers. An example of a double emulsion with Methacrylamide (MAM at 15\%) monomer without cross-linker is given in Figure 1. The globule diameter was around $15 \mu \mathrm{m}$.

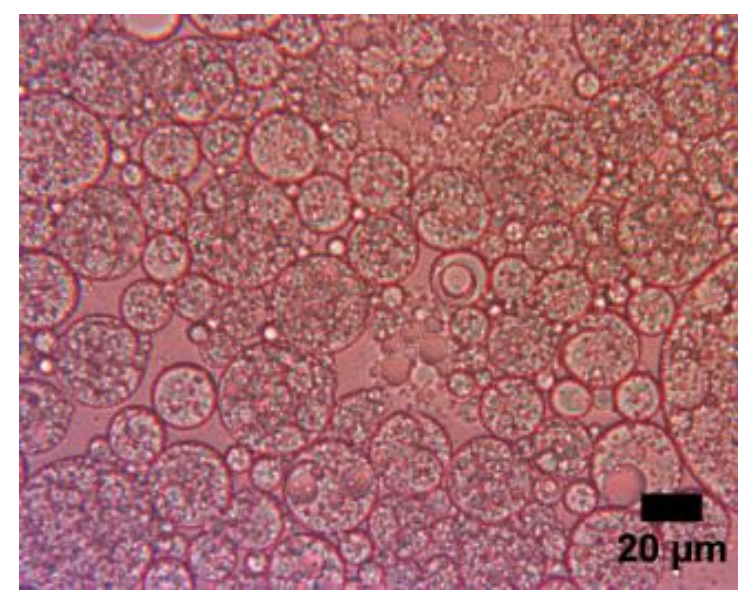

a)

\begin{tabular}{ccc} 
Outer phase & MIP & $22.5 \mathrm{~g}$ \\
$25 \mathrm{~g}$ & PGPR & $2.5 \mathrm{~g}$ \\
\hline Intermediate & Water & $46.15 \mathrm{~g}$ \\
phase & HS & $4.50 \mathrm{~g}$ \\
$60 \mathrm{~g}$ & Monomer MAM & $9.00 \mathrm{~g}$ \\
& Cross-linker & 0 \\
& NaCl 0.1 M & $0.35 \mathrm{~g}$ \\
& KPS & 0 \\
\hline Inner phase 15 & Fragrance & $15 \mathrm{~g}$ \\
$\mathrm{~g}$ & &
\end{tabular}

b)

Figure 1: a) Observation by optical microscopy of the resulting double emulsion with $15 \mathrm{wt} \%$ of MAM with respect to the aqueous phase. Adapted from [33] b). Complete composition of the double emulsion per $100 \mathrm{~g}$ of sample.

II.3 Polymerization of the double oil-in-water-in-oil emulsion

All the emulsions were polymerized in the same way. The polymerization was proceeded in a double envelope reactor with a mechanical stirrer thanks to a motor at a speed of $100 \mathrm{rpm}$. Using the dimensions of the reactor, the speed corresponded to a shear rate of 25 
$\mathrm{s}^{-1}$. The first step was the bubbling of nitrogen for one hour to eliminate the oxygen in the medium. Then, the polymerization was initiated by increasing the temperature up to $70^{\circ} \mathrm{C}$. Temperature and stirring rate were kept constant during 7 hours and the polymerization was stopped with exposure to air. It is worth noticing that the polymerization duration has not been optimized but has been chosen to ensure the most complete polymerization (saturation of the polymerization kinetics). Of course, in the case of a large production, the polymerization time should be optimized i.e. reduced to its minimal value for costs and energy saving reasons. In order to characterize the reaction, the obtained capsules were purified as described below to recover the polymer and to analyze it.

Purification consisted in several washings with ethanol (good solvent of all the reactants to be removed) by centrifugation during $20 \mathrm{~min}$ at $6000 \mathrm{~g}$ (where $\mathrm{g}$ is the gravity constant equal to $\left.9.81 \mathrm{~m} . \mathrm{s}^{-2}\right)$. The obtained solid was then lyophilized.

The global conversion was determined thanks to gravimetric measurements using relation (1):

$$
\text { Conversion }=\frac{\mathrm{m}_{\text {obtained polymer } \times \%_{\text {solid ATG }}}}{\%_{\text {monomer }} \times \mathrm{m}_{\text {collect }}}
$$

$\mathrm{m}_{\text {obtained polymer }}$ was determined by weighting the purified and dried polymer, subtracting the non-volatile substances (surfactants and salt), $\mathrm{m}_{\text {collect }}$ represents the quantity of polymerized medium weighted before purification, $\%_{\text {monomer }}$ represents the initial weight percentage of monomer initially present in the sample and $\%_{\text {solidATG }}$ takes into account the possibility of remaining water despite the lyophilization (and was determined by thermal gravimetric analysis). 
The concentration of N,N-Methylene-bis-Acrylamide (MbA) for this polymerization was fixed at $5 \mathrm{~mol} \%$ compared to Methacrylamide (MAM) that was present at $15 \mathrm{wt} \%$ in the aqueous phase. In other words, if we considered $100 \mathrm{~g}$ of sample, the composition is the following (expressed in grams):

\begin{tabular}{|c|c|c|c|c|c|c|c|c|}
\hline \multicolumn{2}{|c|}{$\begin{array}{c}\text { Outer phase } \\
25 \mathrm{~g}\end{array}$} & \multicolumn{5}{c|}{ Intermediate phase } & $\begin{array}{c}\text { Inner phase } \\
15 \mathrm{~g}\end{array}$ \\
\hline IPM & PGPR & $\mathrm{NaCl}$ & $\mathrm{KPS}$ & $\mathrm{MAM}$ & $\mathrm{MbA}$ & $\mathrm{HS}$ & $\mathrm{H}_{2} \mathrm{O}$ & Fragrance \\
\hline 22.5 & 2.5 & 0.34 & 0.07 & 9.00 & 0.81 & 4.50 & 45.28 & 15 \\
\hline
\end{tabular}

\section{II.3.2. Synthesis of Poly(MAM-TEGDA)}

For this system, the concentration of Tetra(ethyleneglycol) diacrylate (TEGDA) was fixed at $20 \mathrm{~mol} \%$ compared to Methacrylamide (MAM). The MAM monomer was present at $15 \mathrm{wt} \%$ in the aqueous phase. In other words, if we consider $100 \mathrm{~g}$ of a sample, the composition was the following:

\begin{tabular}{|c|c|c|c|c|c|c|c|c|}
\hline \multicolumn{7}{|c|}{$\begin{array}{c}\text { Intermediate phase } \\
60 \mathrm{~g}\end{array}$} & $\begin{array}{c}\text { Inner phase } \\
15 \mathrm{~g}\end{array}$ \\
\hline IPM & PGPR & $\mathrm{NaCl}$ & KPS & MAM & TEGDA & HS & $\mathrm{H}_{2} \mathrm{O}$ & Fragrance \\
\hline 22.5 & 2.5 & 0.34 & 0.07 & 9.00 & 6.39 & 4.50 & 39.70 & 15 \\
\hline
\end{tabular}

\section{II.3.3. Synthesis of Poly(OEGMA-TEGDA)}

The concentration of Tetra(ethyleneglycol) diacrylate (TEGDA) was varied from 5 mol\% to $10 \mathrm{~mol} \%$ with respect to Oligo(ethyleneglycol) methacrylate (OEGMA) and the amount of OEGMA was $25 \mathrm{wt} \%$ with respect to the aqueous phase. So, for 5 and $10 \mathrm{~mol} \%$ of TEGDA, if we consider $100 \mathrm{~g}$ of a sample, the composition was the following.

\begin{tabular}{|c|c|c|c|c|c|c|c|c|}
\hline \multicolumn{2}{|c|}{$\begin{array}{c}\text { Outer phase } \\
25 \mathrm{~g}\end{array}$} & \multicolumn{5}{c|}{$\begin{array}{c}\text { Intermediate phase } \\
60 \mathrm{~g}\end{array}$} & $\begin{array}{c}\text { Inner phase } \\
15 \mathrm{~g}\end{array}$ \\
\hline IPM & PGPR & $\mathrm{NaCl}$ & KPS & $\begin{array}{c}\text { OEGM } \\
\mathrm{A}\end{array}$ & TEGDA & $\mathrm{HS}$ & $\mathrm{H}_{2} \mathrm{O}$ & Fragrance \\
\hline 22.5 & 2.5 & 0.35 & 0.02 & 15.00 & 0.63 & 4.50 & 39.50 & 15 \\
\hline 22.5 & 2.5 & 0.35 & 0.02 & 15.00 & 1.26 & 4.50 & 38.87 & 15 \\
\hline
\end{tabular}




\section{II.3.4. Synthesis of Poly(OEGMA-MbA)}

The concentration of N,N-Methylene-bis-Acrylamide (MbA was varied from $5 \mathrm{~mol} \%$ to $10 \mathrm{~mol} \%$ with respect to Oligo(ethyleneglycol) methacrylate (OEGMA) which was present at $25 \mathrm{wt} \%$ in the aqueous phase. So, for $5 \mathrm{~mol} \%$ (for $10 \mathrm{~mol} \%$ ), if we consider $100 \mathrm{~g}$ of a sample, the composition are given the following:

\begin{tabular}{|c|c|c|c|c|c|c|c|c|}
\hline \multicolumn{7}{|c|}{$\begin{array}{c}\text { Outer phase } \\
25 \mathrm{~g}\end{array}$} & \multicolumn{6}{c|}{$\begin{array}{c}\text { Inner phase } \\
15 \mathrm{~g}\end{array}$} \\
\hline IPM & PGPR & $\mathrm{NaCl}$ & KPS & OEGMA & MbA & HS & $\mathrm{H}_{2} \mathrm{O}$ & Fragrance \\
\hline 22.5 & 2.5 & 0.34 & 0.04 & 15.00 & 1.75 & 4.50 & 38.37 & 15 \\
\hline 22.5 & 2.5 & 0.34 & 0.04 & 15.00 & 3.50 & 4.50 & 36.62 & 15 \\
\hline
\end{tabular}

\section{II.4 Polymer characterization}

The polymers were analyzed by Scanning Differential Calorimetric (DSC) using a DSC Q100 RCS device from TA instruments. Two heating runs were performed. During the first cycle an increase of temperature from $-80^{\circ} \mathrm{C}$ to $120^{\circ} \mathrm{C}$ with a speed of $10^{\circ} \mathrm{C} / \mathrm{mn}$ followed by a decrease down to $-80^{\circ} \mathrm{C}$ was programmed. The second cycle started at $-80^{\circ} \mathrm{C}$ up to $230^{\circ} \mathrm{C}$ with a speed of $10^{\circ} \mathrm{C} / \mathrm{mn}$ followed by a last decrease down to $-50^{\circ} \mathrm{C}$ at the same speed.

II.5 Emulsions and capsules observation

Emulsions and capsules were observed, after dilution in the appropriate solvent, using a Leica Leitz inverted optical microscope and the images were recorded using an IDS video camera and Scion image program. The globule or the capsule size was estimated through a statistic analysis over about 50 globules.

For capsules, scanning electron microscopy (SEM) pictures were captured with a Hitachi TM-1000 apparatus. To avoid deformation or destruction of the capsules by the 
vacuum, when necessary, Cryo-SEM was performed with a JEOL 6700FEG electron microscope equipped with liquid nitrogen cooled sample preparation and transfer units. A drop of capsules dispersion in water was first set on the aluminum specimen holder. The sample was frozen in the slushing station with boiling liquid nitrogen. The specimen was transferred under vacuum from the slushing station to the preparation chamber. The latter was held at $\mathrm{T}=-150^{\circ} \mathrm{C}$ and $\mathrm{P}=10^{-5} \mathrm{~Pa}$ and was equipped with a blade used to fracture the sample. Once fractured, the sample was coated by a layer of Au-Pd and then inserted into the observation chamber equipped with a SEM stage cold module held at $-150^{\circ} \mathrm{C}$.

II.6 Determination of the fragrance localization

\section{II.6.1. Total amount of fragrance}

The capsules dispersions were diluted to $5 \mathrm{wt} \%$ into ethanol and stirred during $30 \mathrm{~min}$ in an ultrasonic bath, in order to swell the microcapsule and to extract the fragrance. This protocol is the same as the one used for shell microcapsules.

This recovered phase was analyzed with a gas chromatograph (Agilent GC 7890B) equipped with flame ionization detector (FID) coupled with Mass spectrometer 5977A (MS), a split/splitless injector (1:40 split ratio), and a data system (Enhanced ChemStation MSD Chemstation). The detector and injector temperatures were set at $250{ }^{\circ} \mathrm{C}$. A $30 \mathrm{~m} \times 250 \mu \mathrm{m}$ internal diameter capillary column coated with 5\%-phenyl poly(methylsiloxane) $(0.250 \mu \mathrm{m}$ film thickness) (HP-5) was used. The oven temperature was programmed from $50{ }^{\circ} \mathrm{C}(2 \mathrm{~min})$ to $280{ }^{\circ} \mathrm{C}(5 \mathrm{~min})$ at $10{ }^{\circ} \mathrm{C} / \mathrm{min}$. Two microliters of the sample were injected. Fragrance molecules are identified with mass spectroscopy and thanks to an internal standard, it was possible to quantify the different fragrance molecules.

\section{II.6.2. Encapsulation efficiency}


The encapsulation efficiency, EE, was indirectly assessed by measuring the fragrance present in the outer phase in closed vials. The encapsulation was then deduced from the total amount of fragrance following:

$$
\mathrm{EE}=\frac{\text { total fragrance amount-fragrance in the continuous phase }}{\text { total fragrance amount }}
$$

To assess the amount of fragrance in the continuous phase, the capsule dispersion was diluted twice in IPM and shook with roll-on during $20 \mathrm{mn}$. This outer phase was then recovered, diluted in ethanol at $5 \mathrm{wt} \%$ and analyzed with the same GC protocol as just described. These measurements were realized at different time intervals in order to identify the possible fragrance diffusion when capsules were stored in closed bottles.

\section{II.6.3. Determination of the capsules leak}

The capsule leak in the atmosphere was determined by measuring the weight loss at regular time intervals of an initial known mass of capsules (without lid) put into a thermostatically controlled ventilated oven at $20^{\circ} \mathrm{C}$ and $40^{\circ} \mathrm{C}$ during several days. To allow comparison the same tests were performed on water, IPM and non-encapsulated fragrance.

\section{II.7 Mechanical resistance of the capsules}

\section{II.7.1. Crushing under a pestle}

This test consisted in crushing the capsules dispersion in a mortar with a pestle. Then, the capsules dispersion was recovered and dispersed in water to be observed by optical microscopy. This simple test allowed determining the capsules sensitivity against crushing and their possible fracture.

\section{II.7.2. Compression test}


The concept of the proposed strategy in order to estimate the resistance of capsules against pressure and more precisely the fragrance release under compression is the following. A known load was deposited on the capsules to impose a given compression stress with a semi-permeable membrane allowing the passage of the continuous phase but not that of capsules that were therefore compressed. The load was maintained to reach a stationary compressed state and the continuous phase was taken up under compression and analyzed to determine its composition. To realize the experiments, a homemade device was set up (Figure 2). It was constituted of three parts. The first was an empty cup where the capsules were placed. The second part was a hollow piston with a stainless-steel filter of $5 \mu \mathrm{m}$ in circle shaped. The filter allowed only the passage of the IPM with the non-encapsulated fragrance maintaining the capsules at the bottom. The piston was made hollow to allow the phase recovery under pressure. The third part corresponded to the load of different weights to access different pressures: 100 and $150 \mathrm{~g}$ corresponded to $780 \mathrm{~Pa}$ and to $1170 \mathrm{~Pa}$ respectively.

The protocol for the measurement was the following: the capsules were put in the cup and covered by IPM (1:1). The piston was placed on the sample and the weight was deposited on it. The pressure was applied for at least one hour to reach a stationary state. Then the IPM with the possibly released fragrance was recovered maintaining the pressure thanks to the hollow piston. This recovered phase was then analyzed by GC to quantify the possibly leaked fragrance in IPM. 

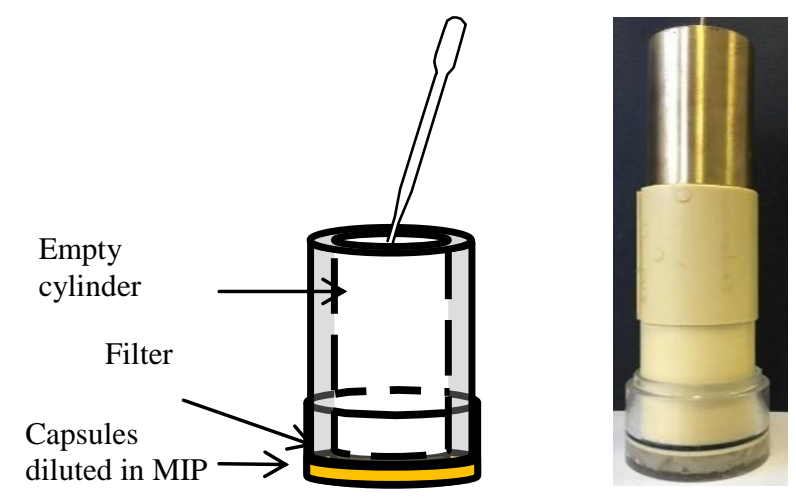

Figure 2: Schematic representation and photography of the homemade device for fragrance release test under compression

\section{Results and Discussion}

Throughout the paper, the double emulsions composition is fixed: $20 \mathrm{wt} \%$ of fragrance droplets inside the globules of the reverse emulsion, the concentration of Tergitol 15-s-12 in water is $7.5 \mathrm{wt} \%$, the concentrations of the mono-functional monomers (Methacrylamide MAM and Oligo(ethyleneglycol) methacrylate OEGMA) are about $15 \mathrm{wt} \%$ and $25 \mathrm{wt} \%$ with respect to the aqueous phase respectively. However, the concentrations of the cross-linkers were varied during the study to highlight its importance in terms of emulsions feasibility or capsules properties.

III. 1 Formulation of the double emulsion with cross-linkers

Two different cross-linkers were chosen: N,N-Methylene-bis-Acrylamide $\mathrm{MbA}$ and Tetra(ethylene glycol) diacrylate TEGDA because of their solubility in water, their non-toxic properties [37] and their reactive entities that are "relatively" similar to the one of the monomers: Methacrylamide MAM and Oligo(ethyleneglycol) methacrylate OEGMA ((meth)acrylic entity). These latter were selected in a previous work [33] because of their biocompatibility, their hydrophilicity and their ability to produce polymer inside an aqueous phase in an inverted emulsion. Thus, 4 couples of monomers and cross-linkers have been studied: MAM/MbA, MAM/TEGDA, OEGMA/MbA and OEGMA/TEGDA 


\section{III.1.1. Formulation of double emulsion with MAM/TEGDA}

The monomer Methacrylamide MAM concentration was about $15 \mathrm{wt} \%$ in the aqueous phase and several concentrations of Tetra(ethyleneglycol) diacrylate TEGDA were tested from $5 \mathrm{~mol} \%$ to $30 \mathrm{~mol} \%$ (Figure 3 ) in order to highlight its effect on the formulation.
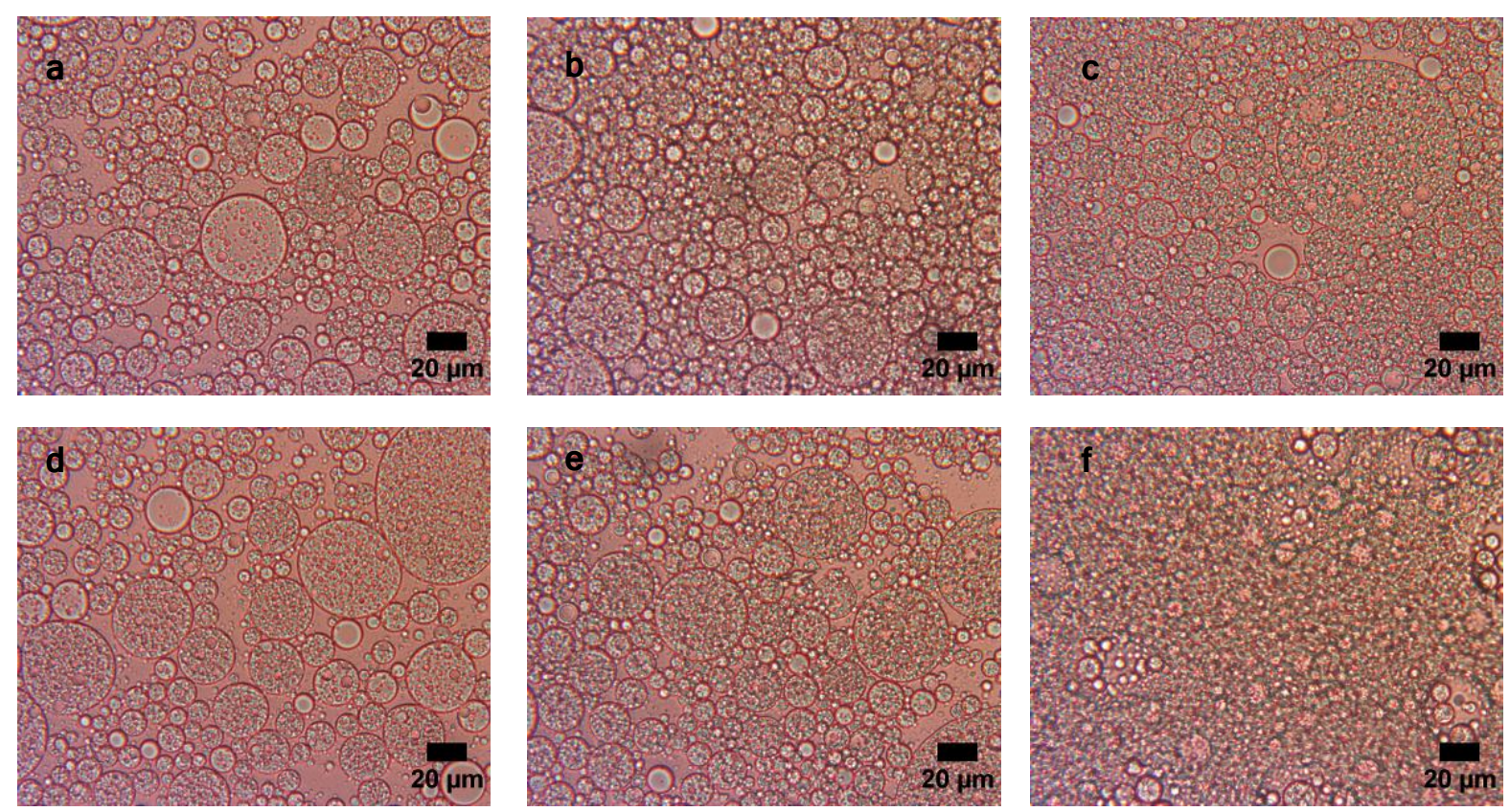

Figure 3: Observations by optical microscopy of the double emulsions containing $15 \mathrm{wt} \%$ of MAM and with various concentrations of TEGDA in the water phase a) $5 \mathrm{~mol} \%$ b) $10 \mathrm{~mol} \%$ c) $15 \mathrm{~mol} \%$ d) $20 \mathrm{~mol} \%$ e) $25 \mathrm{~mol} \%$ and f) $30 \mathrm{~mol} \%$ with respect to MAM.

When the cross-linker was present at a concentration larger than $25 \mathrm{~mol} \%$, the double emulsion was composed of only simple droplets. It is likely due to the ability of the hydrophilic acrylates entities to migrate to the water/IPM interface as it was shown in [33]. Since the cross-linker contains these entities, it might also exhibit a surface activity provoking the destabilization of the double emulsion and leading to simple droplets of inverted emulsion in the medium. On the contrary, when the cross-linker was present at a lower concentration, the double emulsion structure was maintained since the emulsion was only composed of 
multiple drops. So, to get the targeted compartmentalized capsules, the Tetra(ethyleneglycol) diacrylate TEGDA cross-linker concentration had to be lower than the 25 mol\% upper limit.

\section{III.1.2. Formulation of double emulsion with MAM/MbA}

For the N,N-Methylene-bis-Acrylamide MbA cross-linker, only one concentration could be tested for solubility reason. Indeed, for concentration higher than $5 \mathrm{~mol} \%$, the crosslinker was not soluble in water. For $5 \mathrm{~mol} \%$, it was possible to obtain a homogenous in size multiple emulsion as shown in Figure 4.

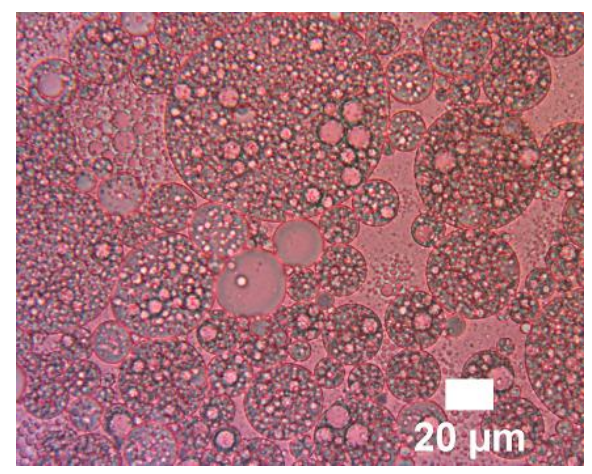

Figure 4: Optical microscopy observation of a fragrance-in-water-in-IPM double emulsion containing $15 \mathrm{wt} \%$ of Methacrylamide and $\mathrm{MbA}$ in water phase. The cross-linker concentration is $5 \mathrm{~mol} \%$ with respect to MAM.

\section{III.1.3. Formulation of double emulsion with the couple OEGMA/TEGDA}

The monomer Oligo(ethyleneglycol) methacrylate OEGMA concentration was set to $25 \mathrm{wt} \%$ in the aqueous phase and several concentrations of Tetra(ethyleneglycol) diacrylate TEGDA from $5 \mathrm{~mol} \%$ to $15 \mathrm{~mol} \%$ were tested (Figure 5). As represented in Fig. 5, the double emulsion structure was preserved up to $10 \mathrm{~mol} \%$. Here again, the acrylate functions of the cross-linker were thought to interact with the oil/water interface leading to the emulsion destabilization. 

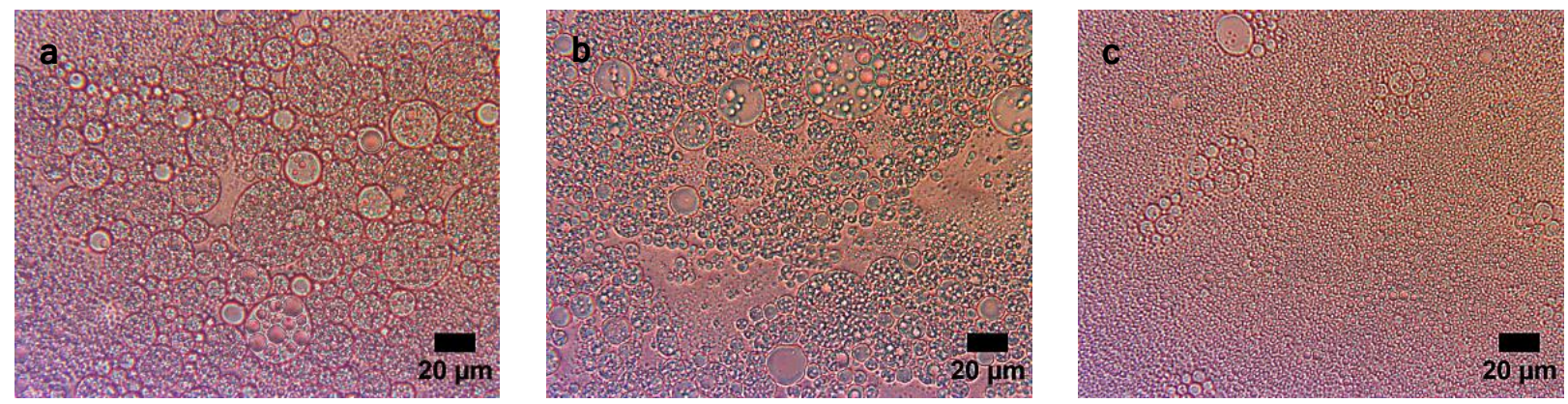

Figure 5: Observations by optical microscopy of the double emulsions containing $25 \mathrm{wt} \%$ of OEGMA in the water phase at different concentrations of TEGDA cross-linker a) $5 \mathrm{~mol} \% \mathrm{~b}$ ) $10 \mathrm{~mol} \%$ c) $15 \mathrm{~mol} \%$ with respect to OEGMA.

III.1.4. Formulation of double emulsion with OEGMA/MbA

The last studied couple was Oligo(ethyleneglycol) methacrylate OEGMA and N,NMethylene-bis-Acrylamide MbA. The concentration of OEGMA monomers in the aqueous phase was also set to $25 \mathrm{wt} \%$ and the concentration of the MbA cross-linker varied from 5 mol\% to 30 mol\% with respect to OEGMA. Micrographs of the resulting double emulsions are reported in Fig. 6.
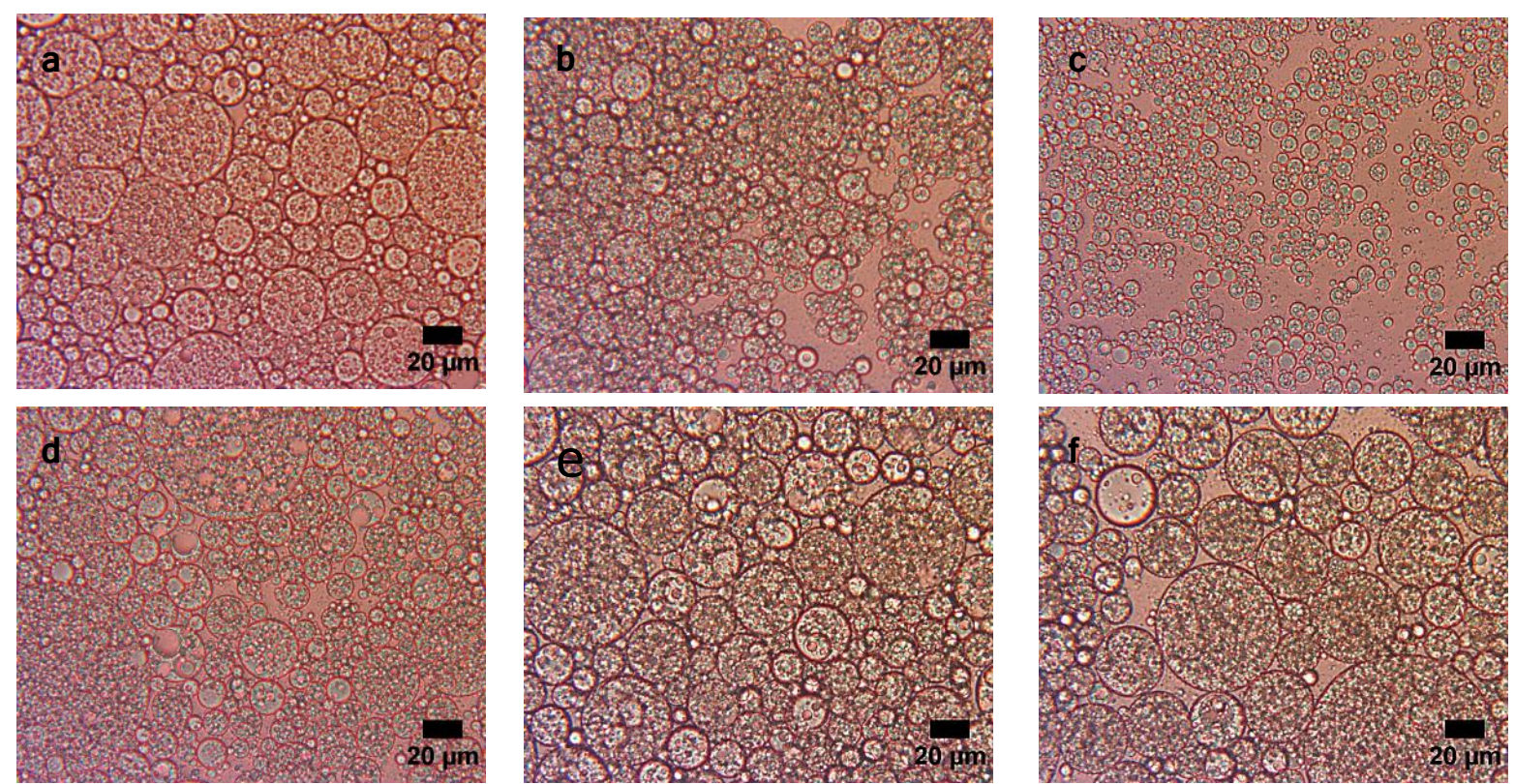

Figure 6: Observations by optical microscopy of the double emulsions formulated with Methacrylamide and TEGDA in the water phase at different concentrations of cross-linkers a) 
$5 \mathrm{~mol} \%$ b) $10 \mathrm{~mol} \%$ c) $15 \mathrm{~mol} \%$ d) $20 \mathrm{~mol} \%$ e) $25 \mathrm{~mol} \%$ and f) $30 \mathrm{~mol} \%$ with respect to OEGMA.

The optical microscopy analysis shows that double emulsions morphology was kept whatever the cross-linker concentration in the studied range (Figure 6).

From this study, it clearly appears that the feasibility of double emulsions depends on the cross-linker and monomer nature and their interfacial properties. The more ability of crosslinker to migrate at the interface, the lower the upper concentration allowing keeping a compartmentalized structure.

III. 2 Polymerization of the double emulsion containing monomers and cross-linkers

\section{III.2.1. Polymerization of the double emulsion with OEGMA/MbA}

Polymerizations were proceeded into a reactor with a mechanical stirrer. They were initiated by increasing the temperature, maintained for 7 hours and stopped with exposure to air. Two concentrations of the cross-linker were studied (5 mol\% and $10 \mathrm{~mol} \%)$. For the two concentrations, polymerization led to two solid samples that could be dispersed in water showing that the capsules kept their individuality. The high solid character arises from the high fraction of capsules (75 wt\%) well above the random close packing. The capsules have to tightly pack and deform. The high compression together with Hertz interactions [38] are likely responsible for the solid observed texture (supporting information SI4). These solids were not dispersible in IPM, which is not a good solvent of the synthesized polymer and induced capsules reversible aggregation (supporting information SI5). On the contrary, the dispersion was possible in water and allowed visualization of the individual capsules. This means that no solvent exchange is necessary for their use or dilution in aqueous phases. Moreover, the obtained capsules had a size very similar to the one of the initial emulsion 
(Figure 7). Note that the fragrance load in the capsules was therefore the same as for the double emulsion that is to say $20 \mathrm{wt} \%$ of the capsules corresponding to $15 \mathrm{wt} \%$ with respect to total sample.
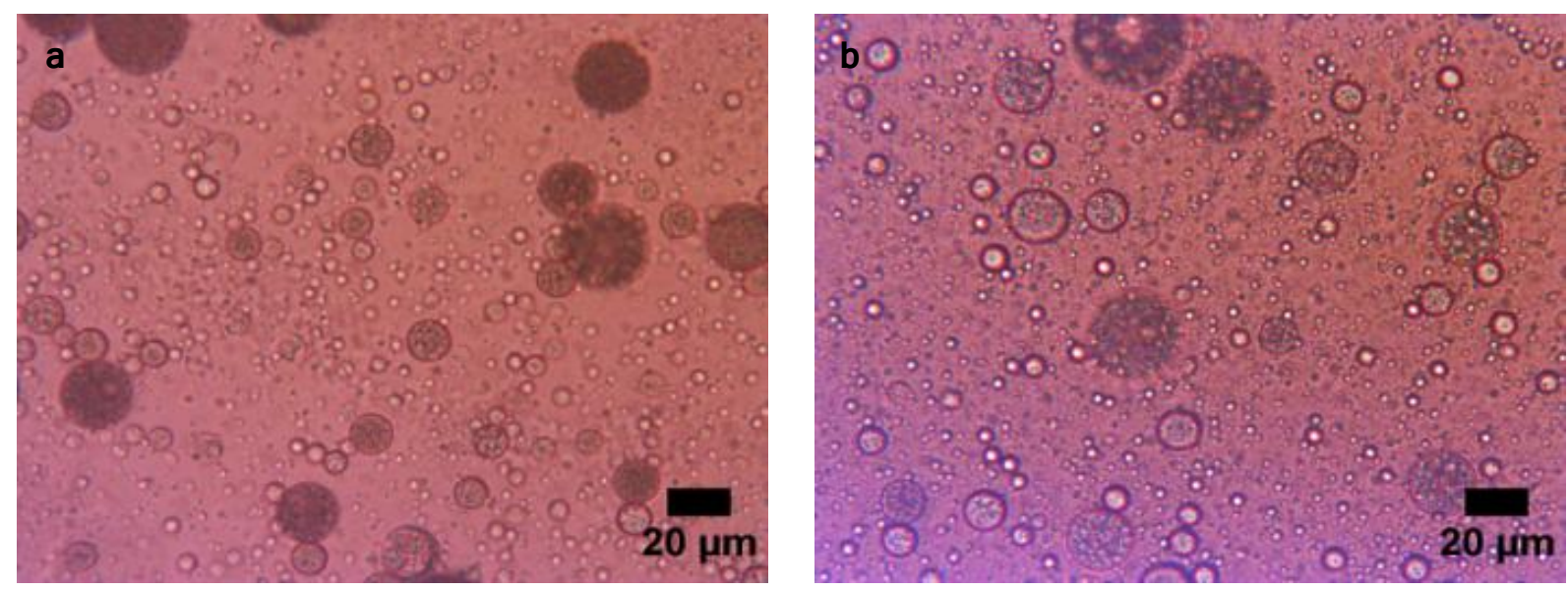

Figure 7: Observation by optical microscopy of the system OEGMA/MbA, with a concentration of $25 \mathrm{wt} \%$ of OEGMA in water, after polymerization and dispersion in water a) capsules obtained from an initial double emulsion containing $5 \mathrm{~mol} \%$ of MbA with respect to OEGMA b) capsules obtained from an initial double emulsion containing $10 \mathrm{~mol} \%$ of $\mathrm{MbA}$ with respect to OEGMA

In addition to the optical microscopy, some SEM observations were performed on the capsules to determine their shape. Capsules prepared with 5 mol\% of MbA (Figure 8a) totally collapsed in the electronic microscope whereas capsules prepared with $10 \mathrm{~mol} \%$ of $\mathrm{MbA}$ (Figure $8 b$ ) remained approximately spherical. This observation already reveals that the two types of capsules exhibit different mechanical resistance under vacuum: higher cross-linking confers the capsules more resistance toward deformation. 

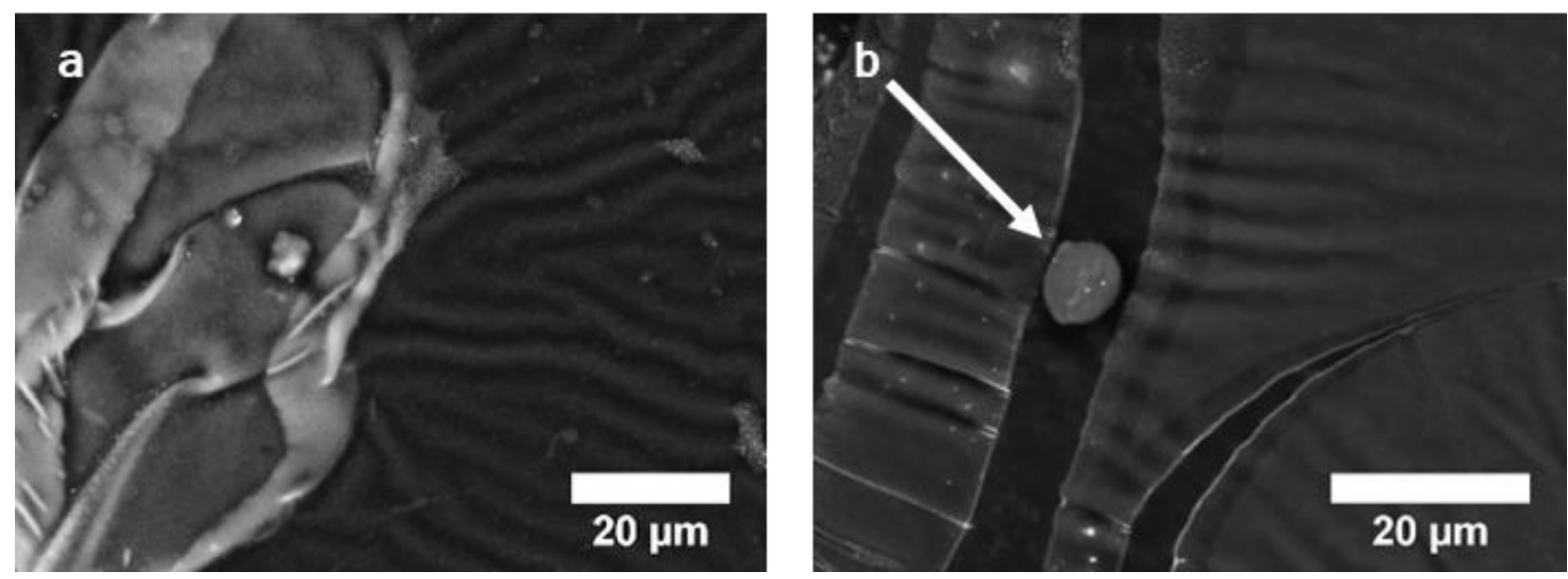

Figure 8: Observation by SEM of the two types of capsules prepared with OEGMA and MbA a) $5 \mathrm{~mol} \%$ of $\mathrm{MbA} \mathrm{b}) 10 \mathrm{~mol} \%$ of MbA. The arrow indicates the spherical capsule.

DSC analysis of the two types of capsules were performed (Figure 9).
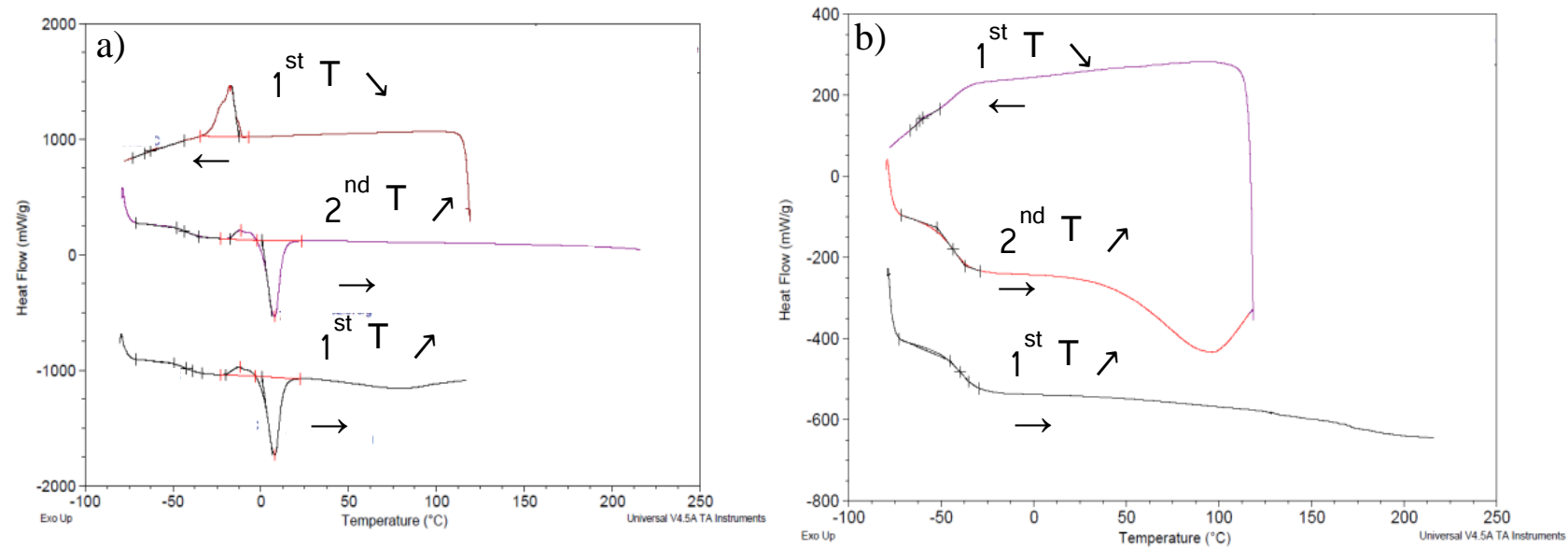

Figure 9: Thermograms obtained by DSC of the two systems containing MbA and OEGMA a) $5 \mathrm{~mol} \%$ of MbA b) $10 \mathrm{~mol} \%$ of MbA after polymerization.

The first observation is the presence of the characteristic signals of the polyethylene glycol (PEG) entities of Oligo(ethyleneglycol) methacrylate OEGMA with a glass temperature transition around $-50^{\circ} \mathrm{C}$, the crystallization around $-7^{\circ} \mathrm{C}$ and the fusion temperature around $5^{\circ} \mathrm{C}$. If we compare these temperatures with those of OEGMA, it can be stated that they are higher, likely as a result of a loss of mobility owing to OEGMA polymerization. Moreover, for capsules containing $10 \mathrm{~mol} \%$ of cross-liker, the crystallization and fusion peaks have 
disappeared. This is due to the mobility decrease of the pendant PEG chains of OEGMA when the cross-linker concentration increases. Indeed, high cross-linking density brings more rigidity to the system; more energy has to be injected to access the same thermo-mechanic behavior.

The global conversions of the polymerization were $83 \%$ and $84 \%$ for the capsules prepared with $5 \mathrm{~mol} \%$ and $10 \mathrm{~mol} \%$ of N,N-Methylene-bis-Acrylamide MbA respectively. These values can be compared to the conversion obtained on the same system (same initial double emulsion) in absence of cross-linker and in a closed flask (35\%) with a magnetic stirring [33]. Therefore, adding the cross-linker and using a reactor boosted the conversion.

III.2.2. Polymerization of the double emulsions prepared with the three other monomer/cross-linkers couples

Similarly, the other couples of monomers and cross-linkers were polymerized and gave promising results. Indeed, polymerization of the MAM/MbA system was proceeded for only one MbA concentration ( $5 \mathrm{~mol} \%$ ) and allowed obtaining capsules with a global conversion of $82 \%$. For the MAM/TEGDA system, we chose to focus on a higher concentration of crosslinker $(20 \mathrm{~mol} \%)$ to combine physical cross-linking due to the MAM with a high chemical cross-linking due to the TEGDA. Capsules were obtained with a global conversion of $81 \%$. For the OEGMA/TEGDA system, several polymerizations were proceeded, and the global polymerization conversion was approximately constant and close to $70 \%$ for both cross-linker ( $5 \mathrm{~mol} \%$ or $10 \mathrm{~mol} \%$ ) concentrations. We therefore decided to keep the capsules with the highest concentration of cross-linker for further characterization to reduce the effect of the absence of physical cross-linking with these compounds. To sum up, there are four different types of capsules at our disposal:

- $\mathrm{MAM} / \mathrm{MbA}$ with 5 mol\% (conversion 82\%) 
- MAM/TEGDA with 20 mol\% (conversion 81\%)

- OEGMA/MbA with 5 mol\% of cross-linker (conversion 83\%)

- OEGMA/TEGDA with 10 mol\% of cross-linker (conversion 73\%)

Note that polymerization did not induce shrinkage or swelling. The size and size distribution width are governed by the initial double emulsion and they are rather well defined compared to other capsules $[39,40]$. As a consequence, the expected initial load of fragrance $(15 \mathrm{wt} \%$ with respect to total sample) is the same for all the capsules. It is also worth noticing that we carefully chose non-ionic stabilizers to avoid any $\mathrm{pH}$-sensitivity. Indeed, for laundry applications for example $\mathrm{pH}$ may vary. As a consequence, on purpose $\mathrm{pH}$ is expected to have no influence on the proposed capsules.

By varying the polymerization chemistry, we expect a diversity of capsules properties. Therefore, we investigated the obtained capsules in terms of morphology, fragrance encapsulation efficiency, fragrance release either in close vials mimicking storage or in contact with air as it may be the case in diverse applications. Capsules are also characterized in terms of mechanical resistance to a compression stress.

III. 3 CryoSEM observation of the obtained capsules

As already seen in Fig. 8, some of the synthesized capsules were sensitive to the vacuum and could not be characterized by SEM (vacuum of the order of $10^{-5} \mathrm{~Pa}$ ). To prevent collapse of the sensitive capsules, we performed Cryo-SEM experiments (Figure 10) that avoid deformation of the capsules due to vacuum and preserve their appearance thanks to a fast sample freezing before introduction in the microscope. 

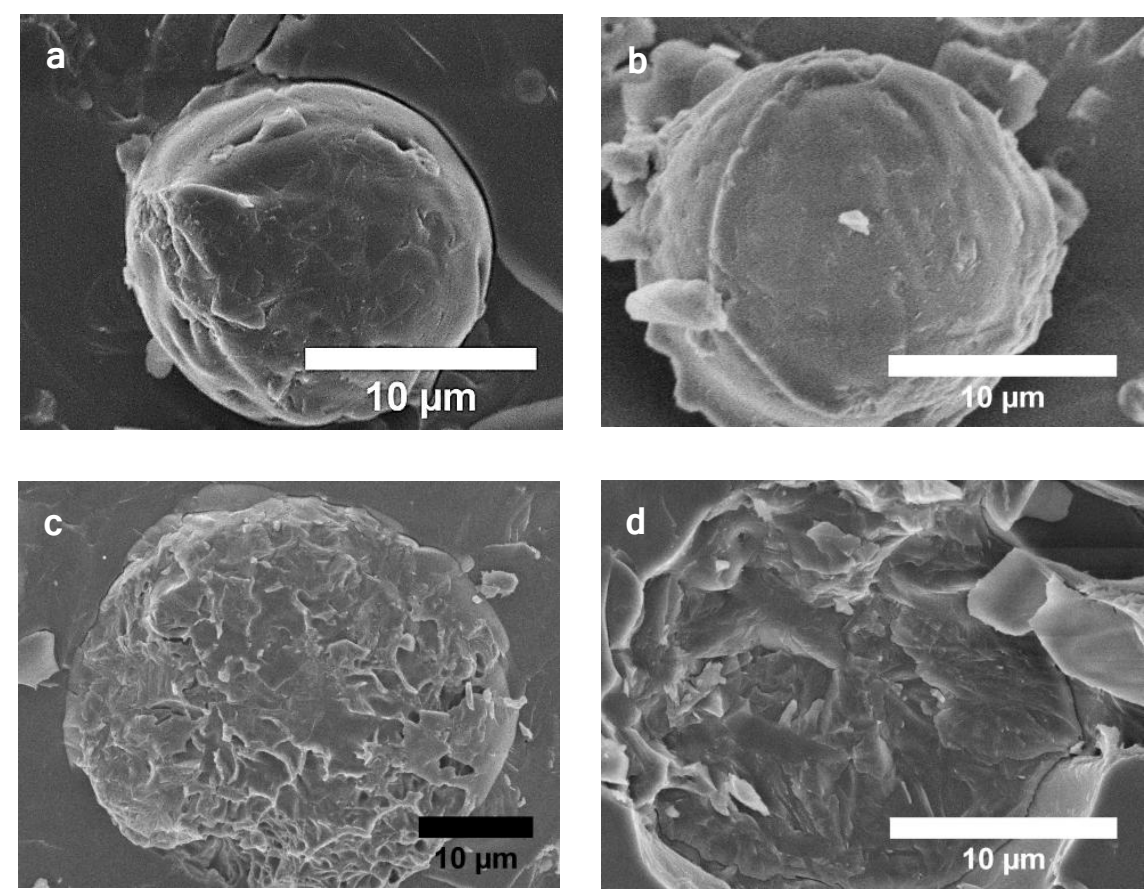

Figure 10: Cryo-SEM microscopy representative pictures of the four types of capsules a) MAM/MbA, b) MAM/TEGDA, c) OEGMA/MbA and d) OEGMA/TEGDA

From Fig. $10 \mathrm{a}$ and $\mathrm{b}$, it can be noticed that capsules containing Methacrylamide MAM (MAM/MbA and MAM/TEGDA) remained intact and exhibited relatively smooth surfaces. This can be explained by the addition of physical cross-linking to the chemical cross-linking, owing to the monomer. This additional cross-linking strengthens the polymer network.

On the contrary, Cryo-SEM images of capsules prepared with Oligo(ethyleneglycol) methacrylate OEGMA (Figure 10: $\mathrm{c}$ and d) reveal an uneven surface and the presence of some small free pieces of solid attesting the fragility of these capsules. In the case of the OEGMA/TEGDA capsules with the highest cross-linker concentration (10 mol\% TEGDA), some broken capsules could be observed (as the one visible in Fig. 10 d). These first observations allowed concluding that capsules formulated with OEGMA were more fragile than the ones with MAM.

III. 4 Localization of the fragrance 
In a second step, we seek measuring the total amount of fragrance (encapsulated or not) after polymerization (Figure 11) in order to determine whether some fragrance has reacted or evaporated during the polymerization or the emulsion preparation step. So, the just polymerized capsules were diluted in ethanol. Ethanol is thought to provoke the fragrance complete leak because it is a good solvent for the fragrance and is able to swell the polymeric capsules. Then, the ethanol phase was analyzed by Gas Chromatography (GC) to quantify the amount of each molecule in ethanol. Assuming that ethanol allowed a complete leak, it corresponds to the total amount of fragrance in each sample.

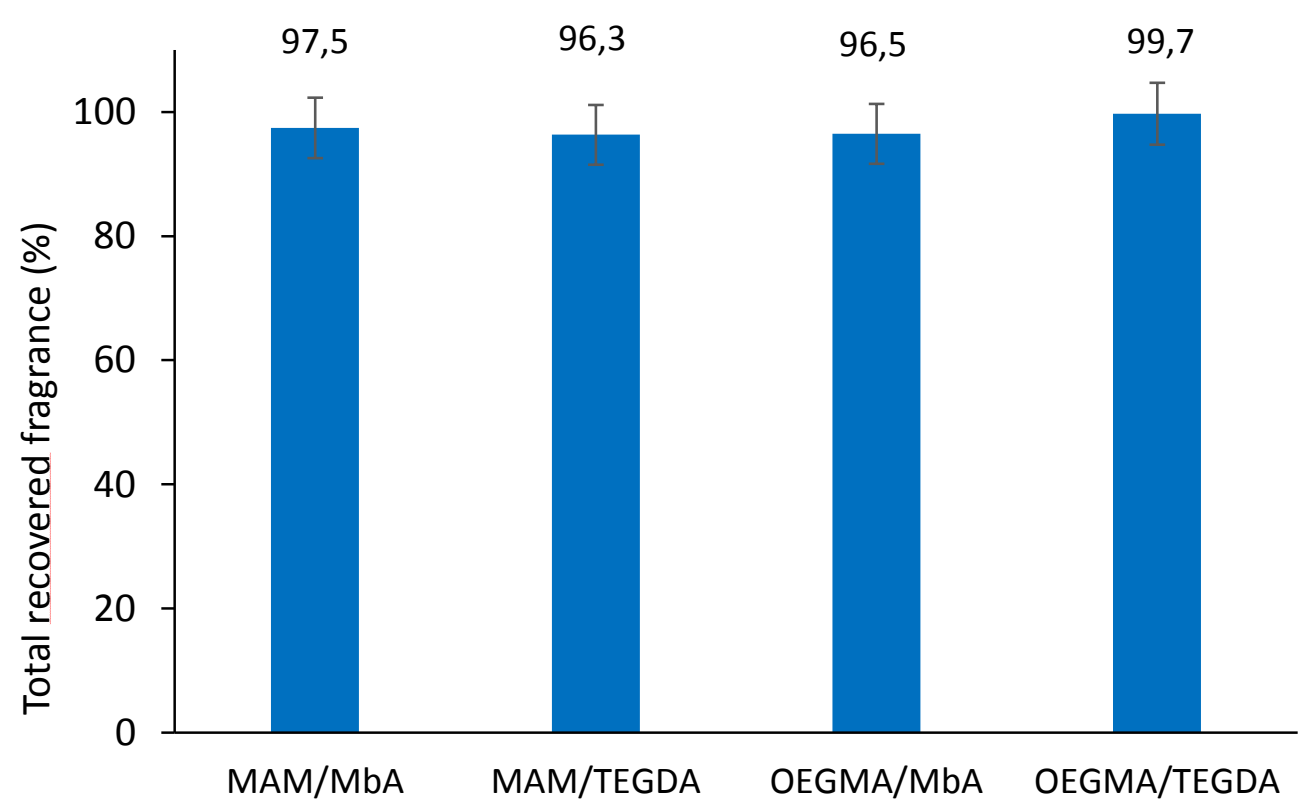

Figure 11: Quantity of fragrance extracted with ethanol and assumed to be the total fragrance contained in the capsule samples. The data are normalized by the initially incorporated fragrance amount. The error bars represent the incertitude of the GC. 
The "total" fragrance recovered for the four capsule families was very close to $100 \%$ attesting that the quasi totality of fragrance stays in the samples. These values are higher than the one determined for migrin oil in melamine resin microcapsules (87\%) [24]. Such high encapsulation efficiencies show that neither a noticeable interaction occurred between the fragrance molecules and radicals, neither thermic degradation, nor evaporation. It can also be concluded that the extraction protocol with ethanol, that was established for core-shell capsules is also globally adapted for the matrix-like capsules.

As a powerful method is used to analyze the fragrance, we can represent the quantity of each fragrance molecule in the extracting ethanol (Figure 12).

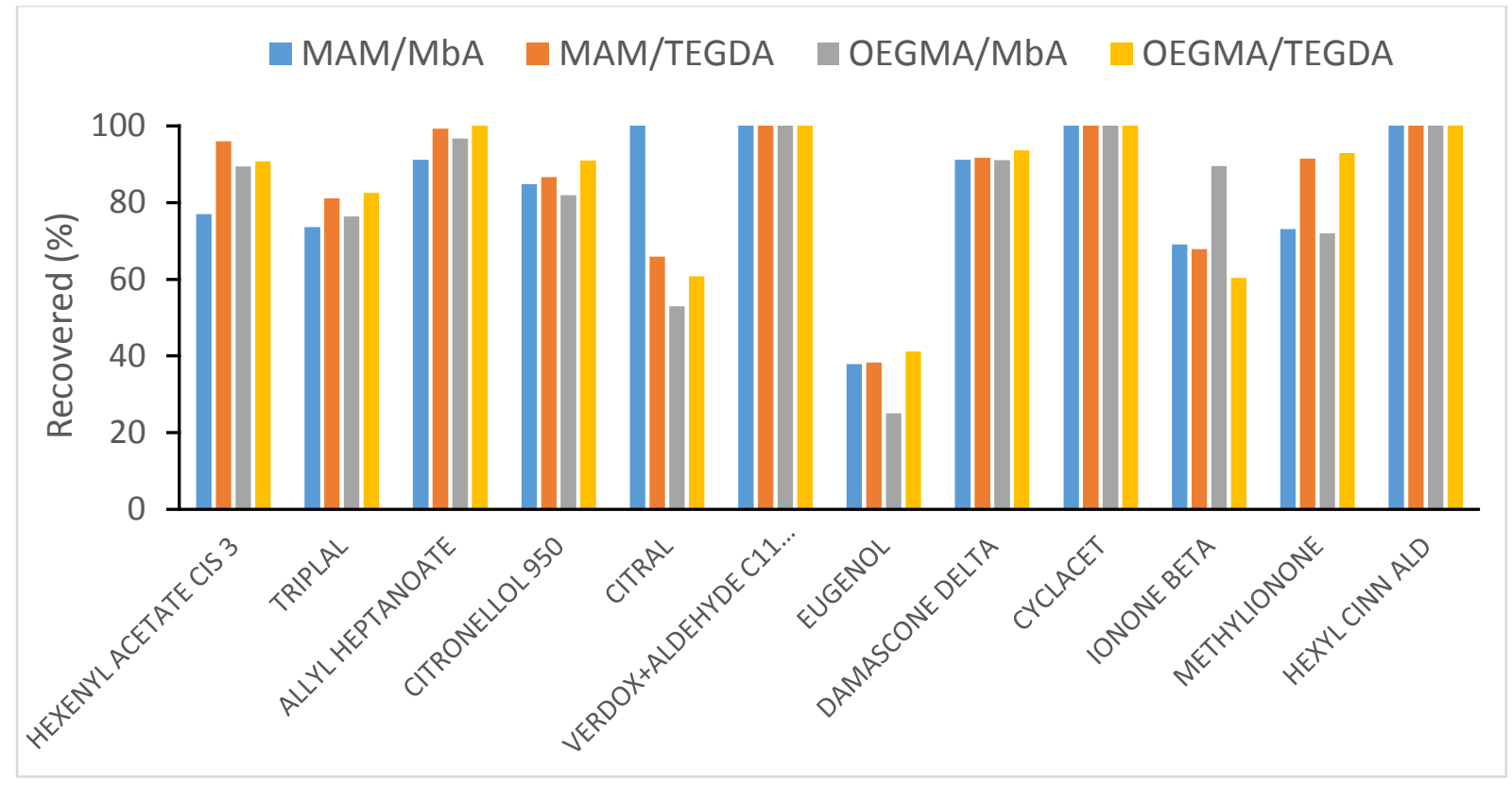

Figure 12: Recovered amount of each fragrance molecules for the four types of capsules For the majority of the compounds, the recovery rates are very high, whatever the polymerization chemistry. However, the recovery rates of eugenol have drastically dropped (lower or close to $40 \%$ ) whatever the considered capsules. The same trend is observed in a lower extend for citral that is poorly recovered (the lowest value is $<60 \%$ ) in three systems among the four tested. The loss of these two molecules can likely not be explained by 
evaporation since their boiling temperature is not lower than that of the other molecules composing the fragrance. In order to determine whether heating or addition of KPS could affect the fragrance composition or amount, we compared the GC analyses of $i$ ) the initial fragrance, $i$ i) the fragrance in contact with water after 24 hours heating at $80^{\circ} \mathrm{C}$ and iii) the fragrance after 24 hours heating at $80^{\circ} \mathrm{C}$ in presence of water (two-phase system) containing 6 mmol of KPS. The results are the following (Figure 13).

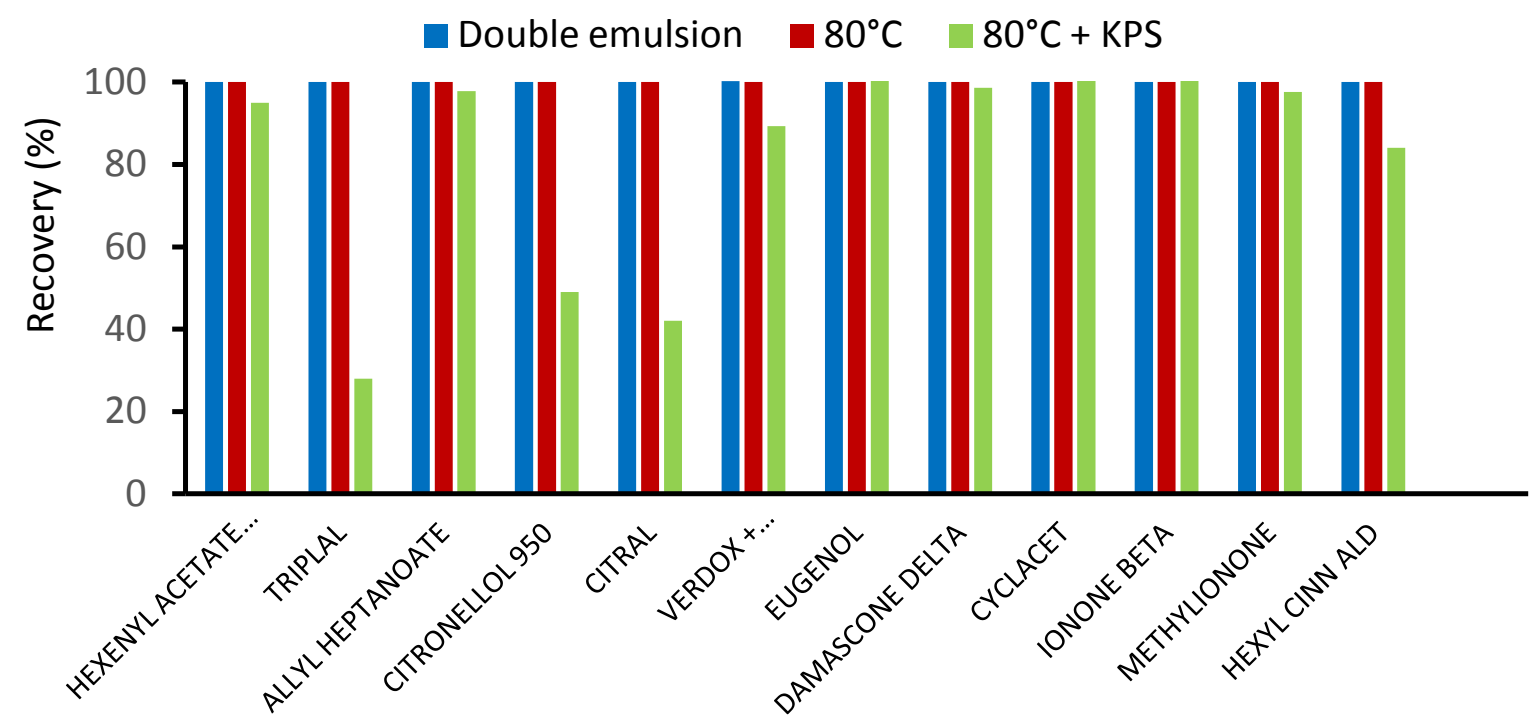

Figure 13: composition of the fragrance in the double emulsion, after heating at $80^{\circ} \mathrm{C}$ and after heating at $80^{\circ} \mathrm{C}$ in contact with water containing KPS.

From Figure 13, it can be seen that encapsulating the fragrance in the double emulsion or heating the fragrance does not modify its composition at all. However if the fragrance is put in contact with water containing KPS, some molecules are highly affected as only a fraction of them are recovered : triplal (28\%), citronellol (49\%), citral $(42 \%)$ and in a much smaller extend hexyl cinn ald (84\%). Comparison between figure 12 and Figure 13 shows that these molecules are much better recovered in the capsules where the water containing KPSfragrance interface is covered by surfactant and in which the water contains water soluble monomers. Therefore, the capsules exhibit a protective action as targeted. It is also interesting 
to note that with this fragrance, no microcapsules could be synthesized through free-radical suspension polymerization, again strengthening the positive impact of the capsules. Figure 13 also shows that eugenol is not prone to chemical alteration as it is recovered at $100 \%$. Therefore, heating in presence of KPS can explain that some citral is missing in the capsules, it cannot explain the loss of eugenol.

In order to get more insight about the parameter responsible for a low retention of eugenol, we plotted the results as a function of the molecules LogP (Supporting Information SI6). These two molecules, citral and eugenol, exhibit among the smallest $\log \mathrm{P}$ values (supporting information SI1) compared to the other molecules composing the fragrance (Supporting Information SI6). Nevertheless, $\log \mathrm{P}$ is not the only parameter that has to be taken into account. Indeed, some molecules exhibit small $\log \mathrm{P}$ values and are totally recovered (Fig. 13) as hexenyl acetate, cyclacet and triplal. Another parameter could interfere, as likely the molecules affinity for the oil-water interface. Thus, in our opinion a way of ensuring a successful encapsulation is to formulate a fragrance composed of molecules characterized by high $\log \mathrm{P}$ values and exhibiting no interfacial properties.

In order to determine if these two molecules are usually difficult to encapsulate, we have compared the encapsulation efficiencies of these two molecules as part of a fragrance in the present case with results on the isolated molecules encapsulated in various systems reported in literature. Compared to other encapsulation systems, even if the encapsulation efficiency of eugenol has drastically dropped, it was higher than system with cyclodetrin $(1.8 \mathrm{wt} \%$ which represent between 80 and $96 \%$ of the eugenol incorporated) [41], nanoemulsion (20 wt $\%$ of the incorporated eugenol) [42] or gelatin-sodium alginate complex coarcevation (16 wt\%) [43]. As far as the Citral is concerned, for its encapsulation in maltodextrin by spray drying, the higher encapsulation efficiency was $16 \mathrm{wt} \%$ (80\% of the incorporated citral in the system)[44] or with Arabic gum by spray drying, the maximum was $8.6 \mathrm{wt} \%$ of citral inside 
the capsules (86\% of the incorporated citral in the system)[45]. Note than in all these systems, no chemical reaction occurred, the loss of encapsulation already resulted from formulation. In our case, the formulation of the double emulsion resulted in $99 \%$ of the total fragrance with no detectable loss of eugenol or citral [32].

As a conclusion, even if all the fragrance molecules might not be recovered at $100 \%$ the proposed novel microcapsule strategy brought advances in the encapsulation ability of a model fragrance composed of 13 molecules.

\section{III.4.2. Evaluation of encapsulated fragrance amount}

The encapsulation fragrance rate was determined by putting the capsules in contact with IPM (Figure 14) in order to only recover the fragrance molecules that are not trapped inside the capsules. Once separated from the capsules, this phase is diluted in ethanol and analyzed by

GC. The encapsulation efficiency is calculated using equation 2

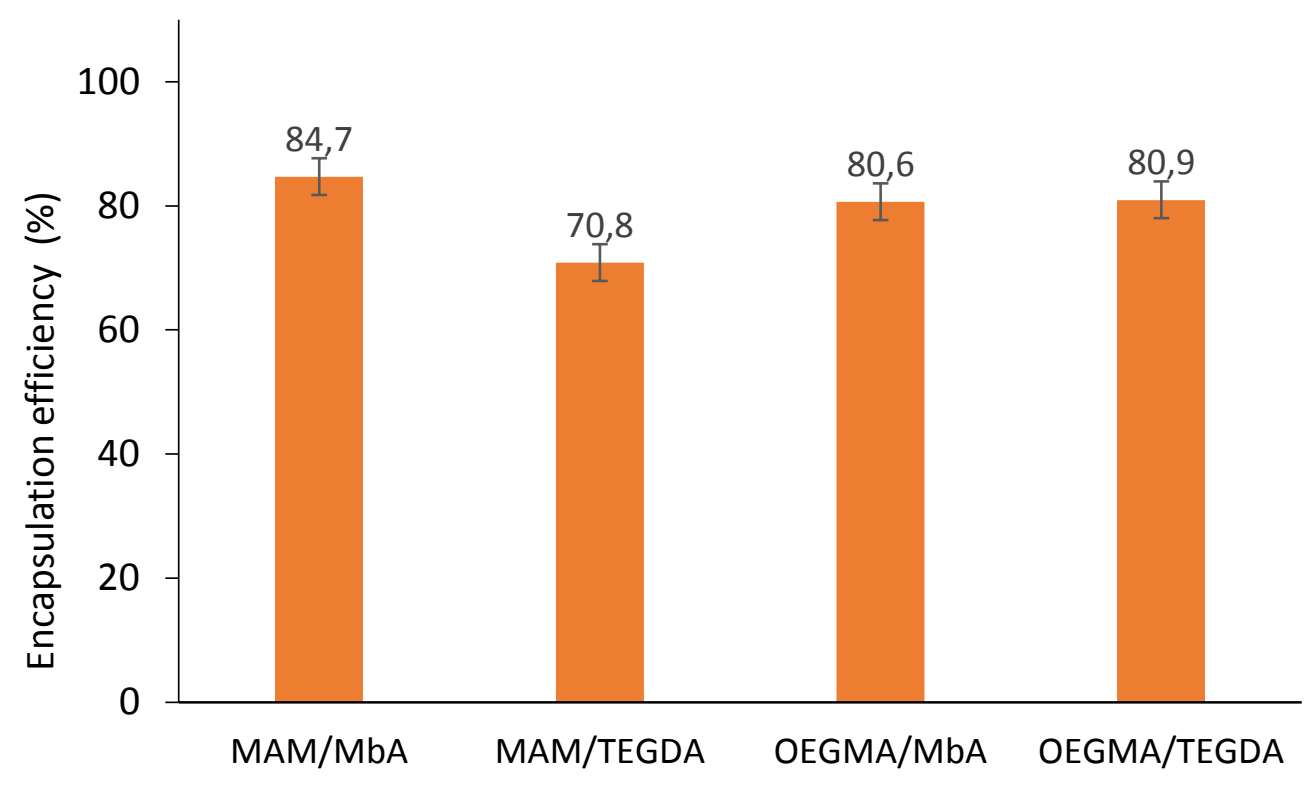

Figure 14: Encapsulation efficiency in the capsule just after polymerization considering the total fragrance and using Equation 2. The error bars represent the incertitude of the GC 
The encapsulation efficiencies just after polymerization were above $70 \%$ for all the capsules types with respect to the initial amount of fragrance. The encapsulation efficiency could be considered quite low compared to the one of oil-in-water core-shell capsules often close to $100 \%$ [46,47], but very high compared to the encapsulation by oil/oil emulsion ( $3 \mathrm{wt} \%$ [41] or compared to oil encapsulation in Arabic gum and maltodextrin by spray drying [48]. However, as the capsules were made from the polymerization of double emulsions, a comparison with these systems is relevant. Two major instabilities are responsible for the release of encapsulated species: compositional ripening and coalescence between a droplet and the globule surface. Compositional ripening corresponds to the diffusion of the innermost oil to the outer oil phase through molecular diffusion in the intermediate aqueous phase or permeation through the interfaces. It is worth noticing that if the double emulsions were completely permeable, fragrance would be released, and IPM would diffuse in the double emulsion to homogenize the composition of the two oily phases. Based on the oily compartment mass repartition, $62.5 \%$ of the fragrance should be leaked in the outer oil that is to say the encapsulation would be at most equal to $37.5 \%$. Indeed for $100 \mathrm{~g}$ of sample, the outer oily phase mass is $25 \mathrm{~g}(62.5 \mathrm{wt} \%$ with respect to total oily phase) and the innermost fragrance mass is $15 \mathrm{~g}(37.5 \mathrm{wt} \%$ with respect to total oily phase). If all the fragrance would permeate, it would be diluted in the outer oily phase leading to $25 \mathrm{~g}$ of outer phase composed of $9.38 \mathrm{~g}$ of fragrance and $15.62 \mathrm{~g}$ IPM and the $15 \mathrm{~g}$ of innermost phase would be composed $5.63 \mathrm{~g}$ of fragrance and $9.37 \mathrm{~g}$ of IPM. As the encapsulation after polymerization is larger than $70 \%$, this shows that compositional ripening did not occur before polymerization. Coalescence events could also have occurred before the polymerization explaining the encapsulation decrease $[49,50]$. Knowing the drop and globule sizes as well as the droplet volume fraction, the number of coalescence events leading to a decrease of the encapsulation by $20 \%$ can be estimated [51]. This corresponds to 135 coalescence events. As polymerization 
was proceeded maximum after 90 minutes, this would lead to a coalescence rate between the fragrance droplets and the aqueous globules of $2.4810^{-3}$ events per minute. This would also mean that a $90 \%$ of fragrance release is expected after 15 and a half hours without polymerization assuming a constant coalescence rate. However, no significant coalescence occurred in 3 days and no such release is seen in the time scale of two weeks. Therefore, in our opinion, coalescence may be ruled out to explain the results.

Again, GC allowed a molecule per molecule analysis, that is reported on Figure 15 and has been determined using Equation 2.

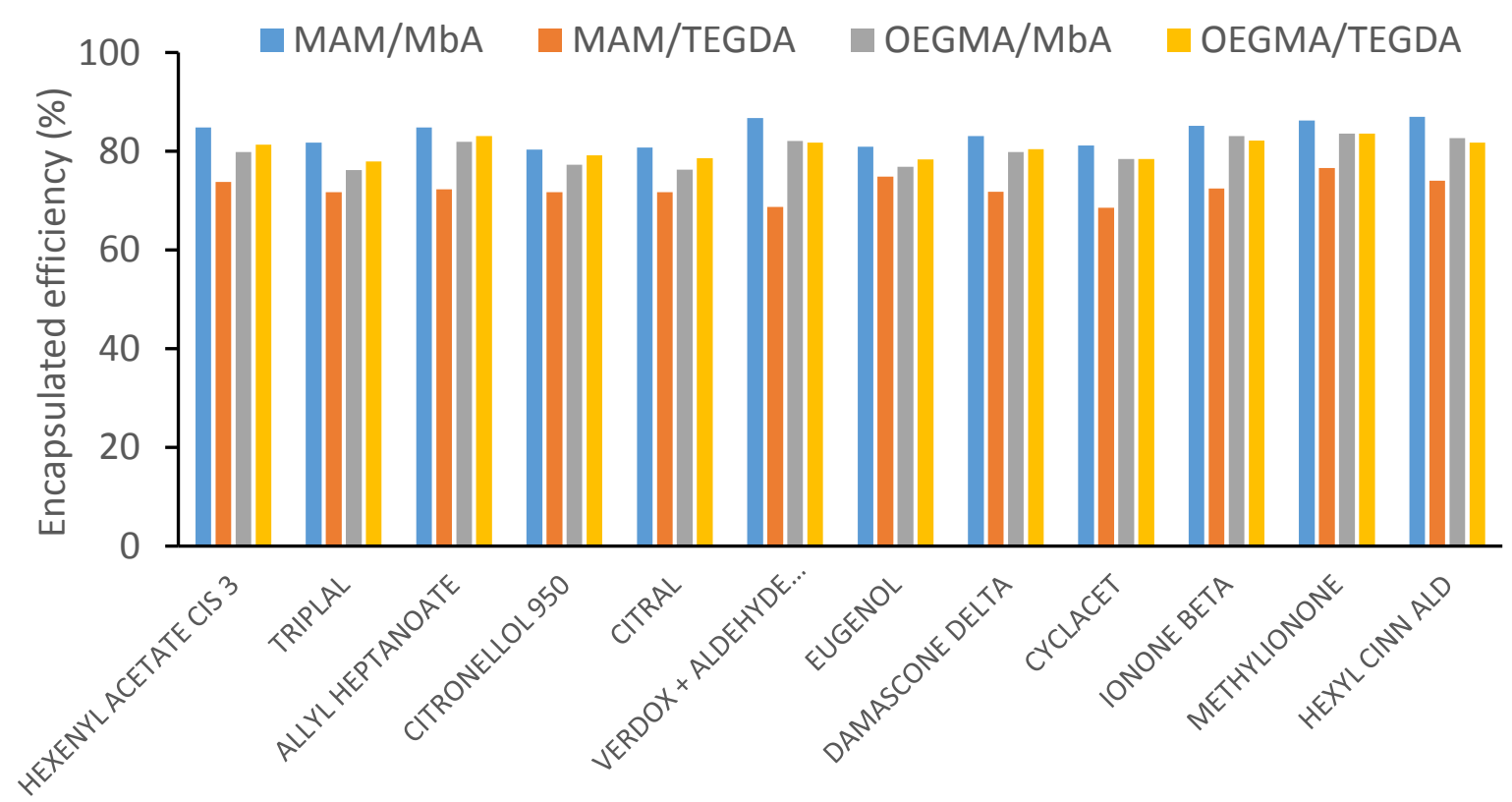

Figure 15: Encapsulation efficiency of each molecule determined by GC and using Equation 2.

\section{III.4.3. Release in closed bottle}

With the same protocol, the release of the capsules in closed bottles was determined at different times (Figure 16). The volume of the vials was equal to $20 \mathrm{ml}$ and the amount of the 
capsules was of the order of $1 \mathrm{~g}$ representing about $5 \%$ of the volume. As the paste of capsules only occupies a small proportion of the closed vials, fragrance is able to escape from the capsules and is not trapped due to the experimental conditions. The capsules are sampled at regular time intervals and diluted in $1 \mathrm{~g}$ of IPM as detailed in the materials and methods section to quantify the amount of remaining fragrance.

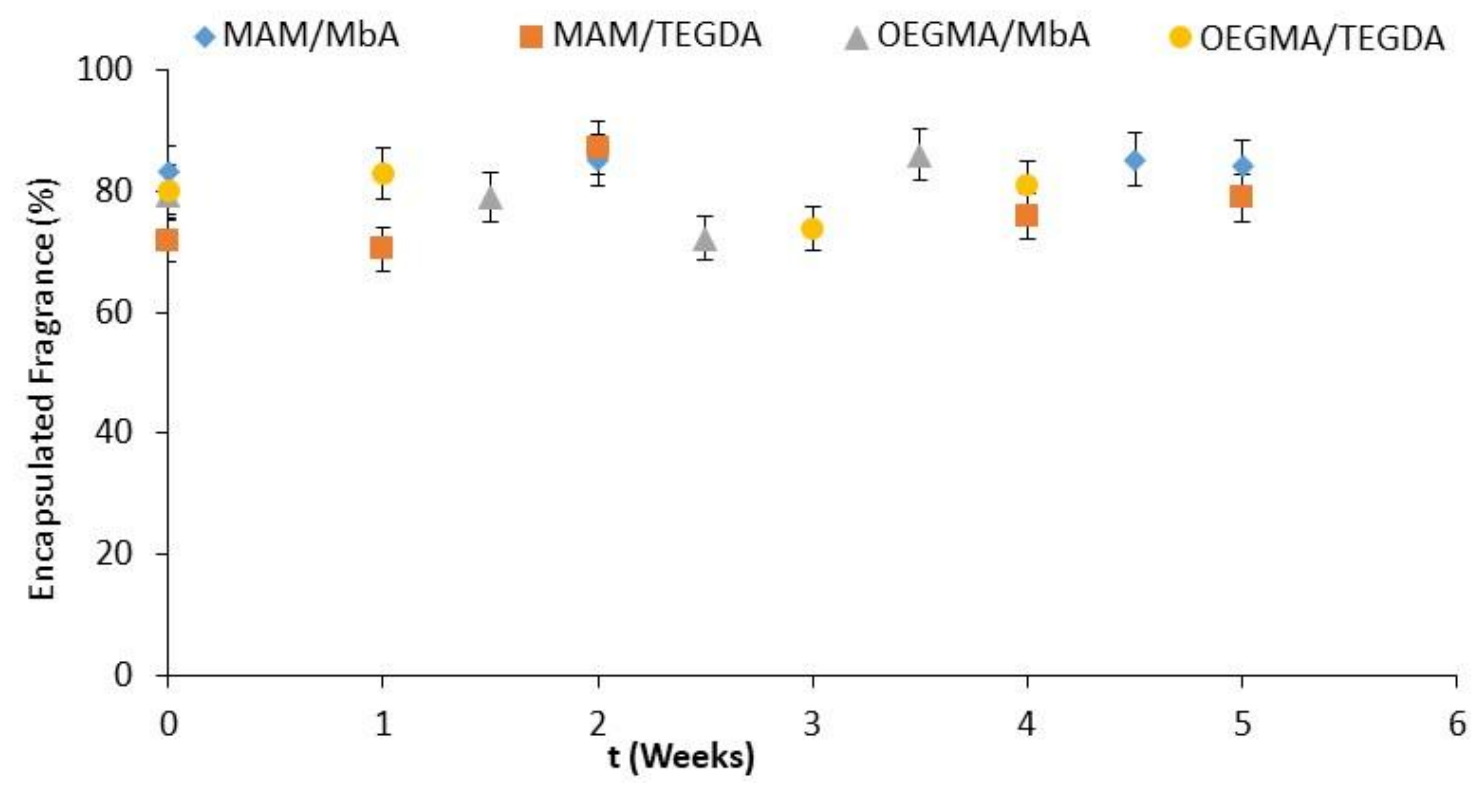

Figure 16: Representation of the evolution of the encapsulated rate over time. The error bars correspond to the incertitude of the GC.

No significant evolution of the encapsulation efficiency could be measured at room temperature as a function of time over 5 weeks (Fig. 16). The amount of encapsulated fragrance remained constant in closed vials, showing the very high stability and efficiency of the four types of capsules. Comparison with double emulsion without polymerization enhances the benefits brought by the polymerization step.

III.4.4. Release properties in open vials 
Another useful property for possible applications is the kinetics of fragrance release in contact with air at room temperature and at $40^{\circ} \mathrm{C}$. This has been measured by weighing the capsules stored in a thermostatic oven at $20^{\circ} \mathrm{C}$ and $40^{\circ} \mathrm{C}$ during several days without lid. The results obtained over 150 days at $20^{\circ} \mathrm{C}$ are reported in Figure 17.

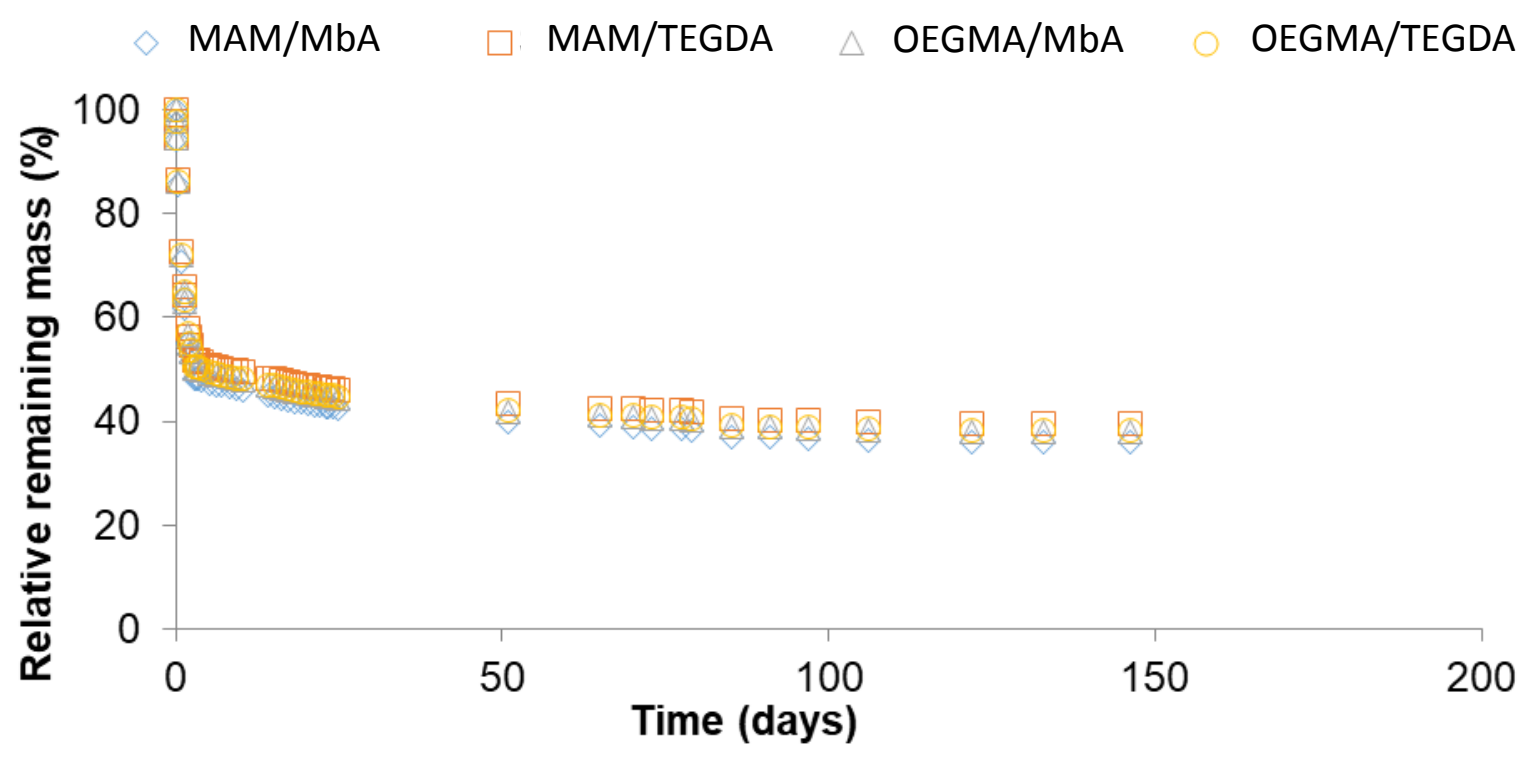

Figure 17: Representation of the time evolution of the capsules weight normalized by the initial capsule weight. The capsules were stored in an open vial at $20^{\circ} \mathrm{C}$.

All the capsules exhibit the same weight loss profile. Indeed, on Fig. 17, a first fast decrease of the weight can be observed during the first three days of the experiments. This decrease can be correlated to the water loss inside the capsules (which represents between 46 and $49.5 \%$ of the weight of the capsules depending on the considered system). The identification with the evaporation of water comes from the fact that water exhibits the lowest boiling temperature. Moreover the same experiments has been reproduced with each of the liquid alone (water, IPM and fragrance: see supporting Information S7). Water was evaporated after 3 days while the amount of IPM did not varied over the all the experimentation duration (150) days. 
Then, after 3 days, a gradual loss was observed up to 150 days. This loss can be attributed to fragrance. Therefore, we can extract the percentage of fragrance remaining in the four capsule families after 150 days (Table 2). Even if the graphs look very similar, real differences between the capsules can be noticed.

Table 2: Summary of the capsule release properties at 150 days at $20^{\circ} \mathrm{C}$ and 40 days at $40^{\circ} \mathrm{C}$, in a temperature-controlled oven. The fragrance loading is the proportion of fragrance remaining in the capsules with respect to the sample mass. Note that initially the loading is 15 $\mathrm{wt} \%$ for all the systems. The encapsulation efficiency is defined by Eq. 2 (it correspond to the proportion of fragrance still encapsulated with respect to the considered initial $15 \mathrm{~g}$ of fragrance for $100 \mathrm{~g}$ of capsules).

\begin{tabular}{|l|c|c|c|c|}
\hline & MAM/MbA & MAM/TEGDA & OEGMA/MbA & OEGMA/TEGDA \\
\hline $\begin{array}{l}\text { Fragrance loading } \\
\text { after 150 days at } \\
20^{\circ} \mathrm{C} \\
\text { (wt\%) }\end{array}$ & 13.9 & 11.2 & 9.1 & 2.7 \\
\hline $\begin{array}{l}\text { Encapsulation } \\
\text { efficiency after } \\
\begin{array}{l}150 \text { days at 20 } \\
\text { (wt } \% \text { ) }\end{array}\end{array}$ & 92.7 & 74.7 & 60.7 & 18.0 \\
\hline $\begin{array}{l}\text { Fragrance loading } \\
\text { after 40 days at } \\
\begin{array}{l}40^{\circ} \mathrm{C} \\
\text { (wt } \%)\end{array}\end{array}$ & 14.3 & 11.8 & 11.2 & 4.0 \\
\hline $\begin{array}{l}\text { Encapsulation } \\
\text { efficiency after } \\
40 \text { days at } 40^{\circ} \mathrm{C} \\
(w t \%)\end{array}$ & 95.3 & 78.7 & 74.7 & 26.7 \\
\hline
\end{tabular}

Depending on the chosen monomer and cross-linker, the range of leakage properties is very wide showing the tenability of the proposed strategy. From the values reported in the tables, it can be notice that the MAM/MbA capsules better retain the fragrance inside the capsules (after 150 days at $20^{\circ} \mathrm{C}$, the system MAM/MbA loses only $8 \%$ of the fragrance. This system is the one that combines chemical and physical cross-linking with the highest proportion of 
physical cross-linking. On the opposite, OEGMA/TEGDA capsules release the highest proportion of fragrance (loss of $82 \%$ of fragrance). Note that they only exhibit chemical crosslinking and have the less dense network.

This allows classifying the capsule families: the retention is higher for MAM than for OEGMA whatever the cross-linker. This can be attributed to the combination of physical and chemical cross-linking leading to denser networks. The difference between the systems can be due to the difference between the reactive entities of the monomer and the cross-linker. For the system OEGMA/TEGDA, which is the one with the most important loss of fragrance, the monomer and the crosslinker are the ones which provokes the obtention of the loosest network. Choosing the monomers and cross-linker therefore allows tuning the fragrance release.

The same experiment was carried out in a thermostatically controlled oven $40^{\circ} \mathrm{C}$ for 40 days. The profile of the weight loss is very similar for the four types of capsules (Supporting Informations S8 and S9). At this temperature, IPM also evaporates. Taking into account the loss of IPM, the amount of fragrance can again be estimated. The results are reported in Table 2. As expected, the temperature provoked the acceleration of the weight loss of fragrance (by a factor close to 3). The proportion of fragrance inside the sample had the same magnitude after 40 days than after 150 days at $20^{\circ} \mathrm{C}$. Indeed, for the MAM/MbA after 40 days at $40^{\circ} \mathrm{C}$, $95.3 \mathrm{wt} \%$ of fragrance remained inside the capsules whereas after 150 days at $20^{\circ} \mathrm{C}, 92.7 \mathrm{wt} \%$ of fragrance remained in the capsules. The same behavior was observed for the other families of capsules and the same tendency was observed.

To conclude, the four systems of capsules exhibit a faster fragrance leakage at $40^{\circ} \mathrm{C}$ than at $20^{\circ} \mathrm{C}$ without changing the classification. Moreover, this study allows classifying the four 
systems from the less to the most fragrance retentive or from the most to the less diffusive systems (Fig. 18):

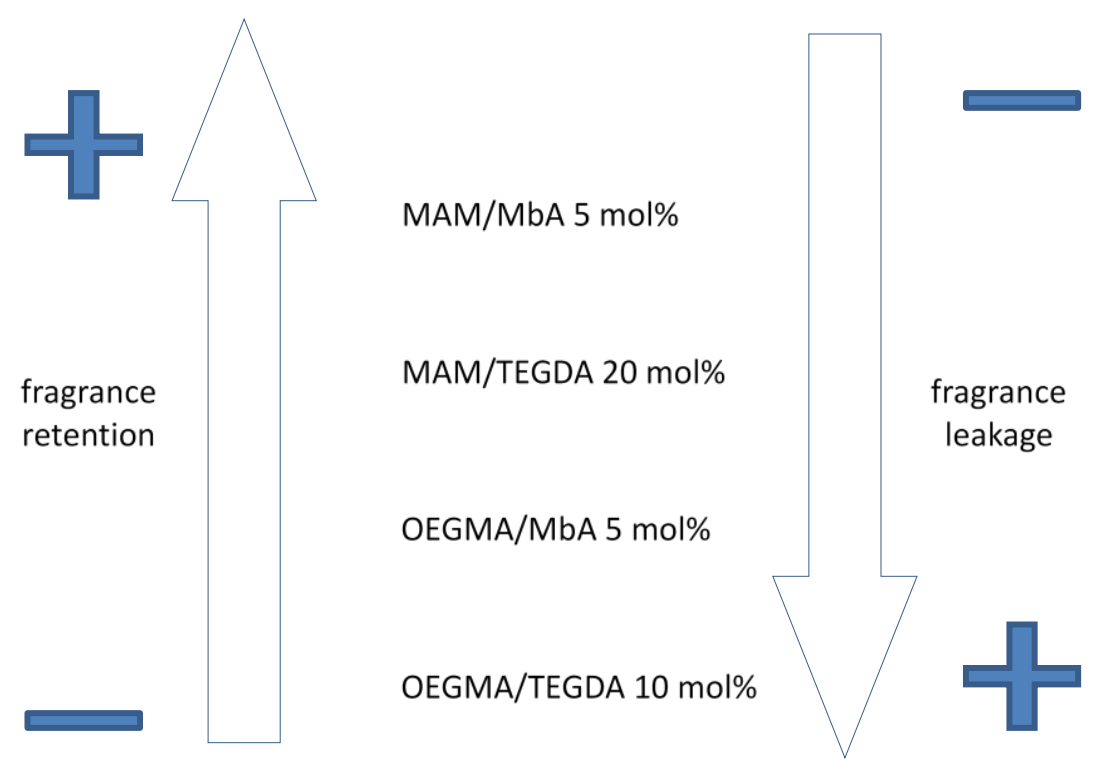

Figure 18: Classification of the different capsules in term of leakage or retention (depending on the desired property).

III. 4 Mechanical properties

In this part the mechanical properties of the four systems was studied. Capsules were crushed with a pestle in a mortar to provoke the release of fragrance and assess their possible rupture. 

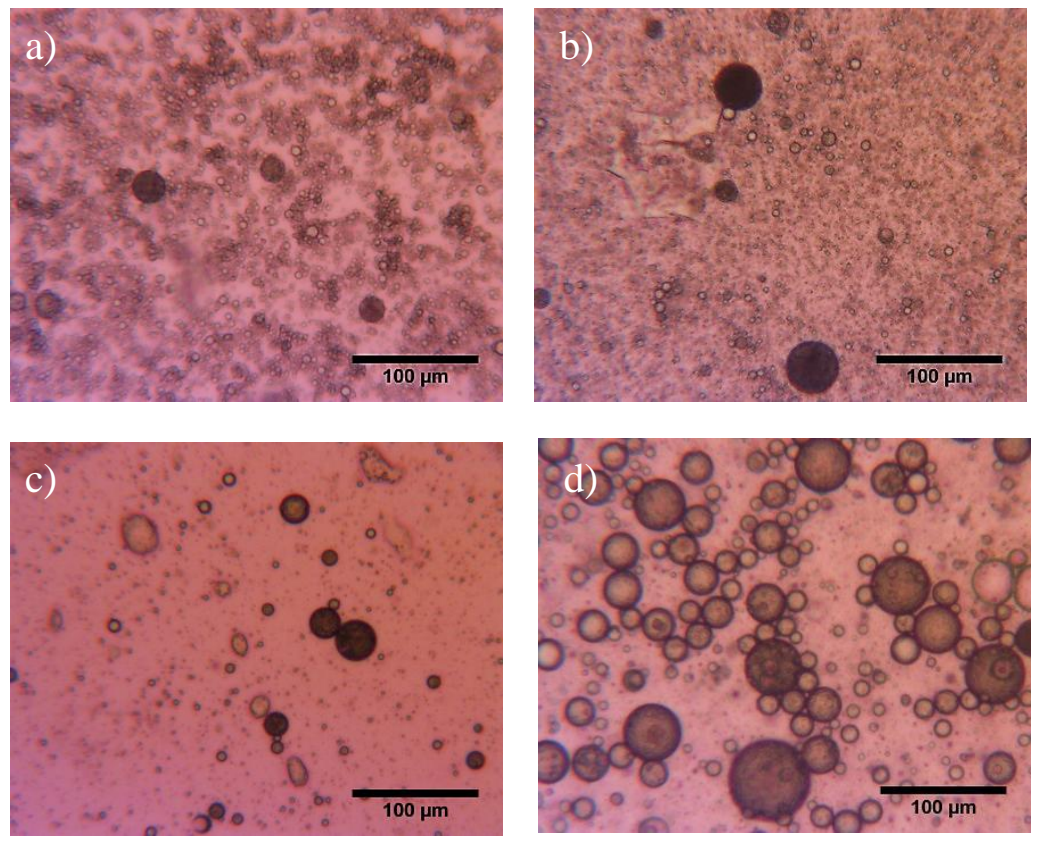

Figure 19: Observations by optical microscopy of the different redispersed in water after the crushing a) MAM/MbA b) MAM/TEGDA c) OEGMA/MbA and d) OEGMA/TEGDA

Capsules were dispersed in water after crushing and observed by optical microscopy. As can be seen in Figure 19, they were all intact, no fragment could be observed. This means that they all sustain a high mechanical stress. Moreover, during the crush, a boost of fragrance could easily be detected revealing a fragrance release. To get further insight into their mechanical resistance to stress, the capsules were submitted to increasing compression using the device described in the materials and methods section. While maintaining the applied pressure, the released liquid could be taken in IPM, diluted in ethanol and analyzed by GC. Alternatively, the pressure could be released and the liquid remaining outside analyzed by GC (Figure 20). 


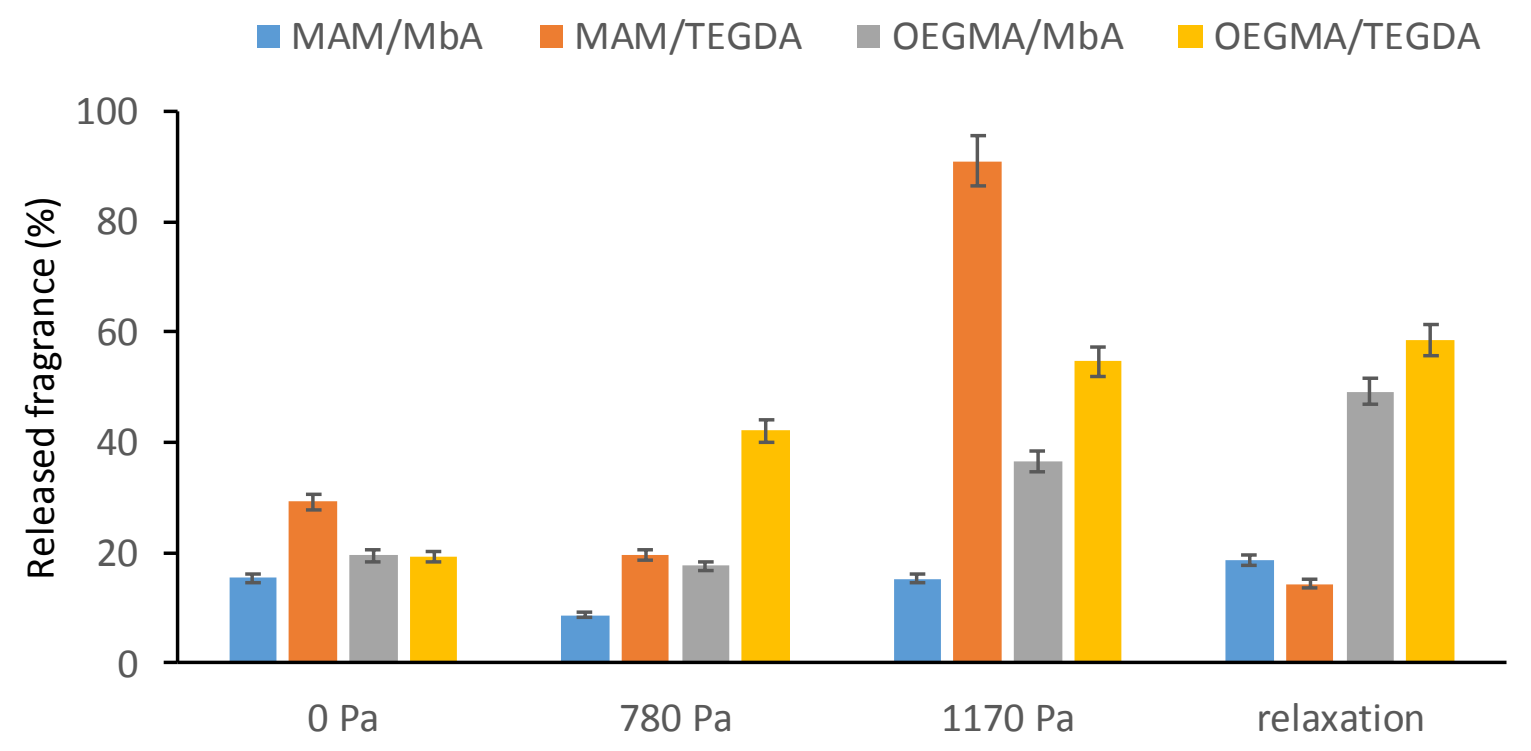

Figure 20: Representation of the percentage of fragrance, with respect to the initial amount of fragrance, recovered in the IPM as a function of the applied pressure i.e. that has been released in the outer phase. Relaxation means that the pressure was released before withdrawing the IPM solution.

From Fig. 20 the general trend observed is that the fragrance release increases with the applied pressure showing that pressure is an efficient trigger. A detailed analysis by molecule is given in Supporting information S10. This means that additionally to the slow and continuous release characteristic of diffusive capsules, they can also be considered as stimuli responsive. Optical microscopy observation of capsules after this experiment showed that no capsule fragment was present, all the capsules were intact. We therefore propose that the capsules behave as small sponges that release their content under pressure without breaking. To test further this hypothesis, we investigated their ability to reabsorb a part of the released content by cancelling the applied highest pressure after 12 hours. The outer oily phase is withdrawn 12 hours after the stress release. The results are reported in Fig. 20 under the 
denomination relaxation. The amount of possibly reabsorbed fragrance is not detectable except for the MAM/TEGDA capsules.

IV. Comparison with some other capsules.

The comparison of the proposed capsules with all the existing ones without focus on the goal and application is just an impossible task in the frame of an original paper. Therefore, the ambitious of this paragraph is limited to a comparison with some recent and novel systems $[19,20,23,39,40,52-63]$ that aims at reducing or avoiding the use of formaldehyde, most of them being core-shell capsules. Also depending on the considered application, either a provoked sudden release of the cargo is aimed at $[19,20,54-56,60-62]$ or, on the contrary, a slow and sustained released is preferred or observed as it was the goal herein $[39,40,52]$. Different strategies have been envisioned among them one can distinguish:

i) pre polymerization of the formaldehyde [23,25], in this case a pre polymer is formed in the continuous aqueous phase and this pre polymer can adsorb or condensate at the emulsion drop surface. The advantage of this protocol is the absence of reactive entity, in the disperse phase except those composing the fragrance. As in our case, the reaction does not take place in the fragrance. The disadvantage of these proposed capsules is the persistence of the presence of formaldehyde despite a lower amount as shown in [25]. Moreover the elaboration of capsules requires the use of concentrated alkaline $(0.5 \mathrm{~N}$ of $\mathrm{NaOH}[23,25])$ and acid solution (0.5 $\mathrm{N}$ acetic [23] or citric acid [25]). In [25] the encapsulation efficiency has been measured between 67 and $81 \%$.

ii) the change of the resin chemistry [13,39]. For example, in [39] formaldehyde is replaced by glyoxal and branched oligomers are first prepared. Unlike melamineformaldehyde capsules for which the release is generally triggered by the rupture of the brittle and stiff shell, the formaldehyde-free capsules are not hermetic and the authors determined a release rate of the order of $2-4 \times 10^{-3} \mathrm{mg} \cdot \mathrm{min}^{-1}$ at $50^{\circ} \mathrm{C}$. Adding in-situ cross-linking in the shell 
allows decreasing the release at the very low value of $0.007 \times 10^{-3} \mathrm{mg} \cdot \mathrm{min}^{-1}$. For comparison, the averaged release rates of our capsules may be converted using the same unit: they range from $5 \times 10^{-3} \mathrm{mg} \cdot \mathrm{min}^{-1}$ (MAM/MbA) to $57 \times 10^{-3} \mathrm{mg} \cdot \mathrm{min}^{-1}$ (OEGME/TEGDA) at $20^{\circ} \mathrm{C}$ and from $12 \times 10^{-3} \mathrm{mg} \cdot \mathrm{min}^{-1}$ to $190 \times 10^{-3} \mathrm{mg} \cdot \mathrm{min}^{-1}$ at $40^{\circ} \mathrm{C}$. Note that in our case, tightness was not the goal as we aimed at proposing capsules with sustained fragrance release.

iii) solvent extraction $[13,52,53]$. In many cases a solvent, that is a low boiling good solvent of the polymer composing the future capsules shell is used and mixed with the fragrance. Then this solvent has to be extracted to allow precipitation of the polymer at the interface. Dichloromethane $[13,53]$ is the most often used solvent that now tends to be replaced by ethylacetate [52]. In this latter article, cellulose or cellulose-silica hybrid capsules are obtained by solvent evaporation and consequently phase separation occurs between the oil phase and cellulose-silica. The release of lavender is assessed with and without silica. After 30 days, $47.8 \%$ and $70 \%$ respectively of lavender has been released at $20^{\circ} \mathrm{C}$ and $40^{\circ} \mathrm{C}$ respectively while the addition of silica decreased the release to $25.3 \%$ at $20^{\circ} \mathrm{C}$ and to $45 \%$ at $40^{\circ} \mathrm{C}$.

iv) interfacial polymerization is at the origin of the shell formation of capsules in [40]. The $15 \mu \mathrm{m}$-sized capsules contain equal weight mixture of cyclosal, romascone, verdox and dorisyl. The oil phase contains an acrylate monomer for "click" chemistry while the aqueous phase contains dithiol, an emulsion stabilizer and $\mathrm{K}_{2} \mathrm{CO}_{3}$ as catalyst. Varying the proportion of dithiol and acrylate allows varying the encapsulation efficiency from 91 to $96 \%$ just after encapsulation to $83-92 \%$ after one month at room temperature and $80-86 \%$ after one month at $45^{\circ} \mathrm{C}$. As for previous capsules, a reactive monomer has to be introduced in the fragrance and the release rate vary in a much lower range than the capsules presented herein.

v) Pickering emulsions as templates with sol-gel chemistry or interfacial polymerization. Pickering emulsions that is to say emulsions stabilized by particles are known 
to be very stable kinetically. In order to improve their tightness or mechanical properties the emulsion surface may be reinforce either by polymers that serve as a mortar [55], by polymerization at the interface [57] or by in-situ synthesis of a silica shell [19,20,54,60-65]. In this case, the goal is to prepare tight capsules for long-term storage with a release provoked by an external field that can be a mechanical stress $[20,57,62]$, a temperature raise $[60,64,65]$, the application of a magnetic field for hyperthermia [61] and so on.

One could discuss whether these Pickering-based capsules belongs to the new class of the so-called colloidosomes $[55,66]$ that are shell-like capsules formed by the self-assembly of colloidal particles at the interface of emulsion droplets. The term "colloidosome" has been proposed by Dinsmore and co-workers [67] and designates such drops covered by colloidal particles with a second step consisting in the particle locking at the interface and a possible third step where capsules are transferred by centrifugation into a solvent that is typically the same as the internal phase. This means that there is no interface between the internal and external fluids and it allows the colloidosome permeability to be controlled by the interstitial holes. A recent example of colloidosomes is given by ref [63]. Indeed in this later paper water-in-oil emulsions are prepared and stabilized by hydrophobic particles that are further locked by the synthesis of a silica shell inside the aqueous drop, before capsules are transferred into an aqueous phase. The capsules are considered as tight by the authors (10\% of release after 80 hours) and the release of methyl blue as a model drug is provoked by addition in the outer phase of alcohol or surfactant.

vi) use of a triple emulsion. In a recent paper, Lee and coworkers [56] propose to produce an $\alpha$-pinene (a water soluble fragrant molecule)-in-oil emulsion via bulk emulsification. Then, this reverse emulsion is co-flowed using microfluidics with a photocurable oil before both are flow-focused by an aqueous continuous phase and photopolymerized using UV exposure to produce polymeric $200 \mu \mathrm{m}$-sized microcapsules with 20 
$\mu \mathrm{m}$ thick shells. The aqueous phase may also contain a photo-curable hydrophilic poly(ethylene glycol) diacrylate (PEG-DA) allowing to get a hydrogel and therefore to enhance the retention. After 5 days, about $40 \%$ of $\alpha$-pinene remained encapsulated at room temperature while the presence of hydrogel allowed increasing it to 50\%. In the present paper, after 150 days that is to say a period 30 times longer, the values varied from $13 \%$ to $97 \%$ depending of the type of capsules. Capsules made from the triple emulsion are of very large sizes and are highly monodisperse due to the use of microfluidics. Note that when PEG-DA is used, the polymerization again takes place in the compartment containing the fragrant hydrophilic molecule.

Another point that can be mentioned is the fact that most often the encapsulated species is a unique and model molecules used to represent a fragrance $[23,52-54,56,60,63]$. Only few papers reports the use of a blend of four or up to six molecules $[39,40,57,58]$ and even less consider real fragrances [13].

This short state of the art allows summarizing the advantages of the herein proposed capsules based on the polymerization and cross-linking of a double emulsion intermediate phase:

- polymerization does not take place at the fragrance location

- there is no need of an additional solvent that has to evaporate

- there is no need of scavenger

- there is no need of a catalyst

- there is no need of transfer

- polymerization does not require hard acid or alkaline conditions

- the proposed cargo is a real fragrance composed of 13 molecules classically used in perfumery 
- the cargo is composed of $100 \%$ of fragrance

- capsules have reasonable size distribution widths despite the simple stirring conditions

- capsules exhibit a sustained release that can be judiciously tuned in a very large range

- additionally to the sustained release, a mechanical provoked release can be imposed

- capsules do not break even after provoked release and behave as small sponges meaning that a fragrance reload is possible.

\section{Conclusion}

We could synthesize four systems of capsules able to encapsulate a large amount of a real and model fragrance, which is a mixture of reactive ingredients. The capsules are based on the elaboration of fragrance-in-water containing hydrophilic monomers and cross-linkersin-perfumery oil double emulsions and on the polymerization of the intermediate aqueous phase. This is a real breakthrough in capsule elaboration science through polymerization that generally focuses on the polymerization of, either the dispersed phase of a simple emulsion or the interface. In both cases, monomers are most often present in the same phase as the fragrance composed of reactive molecules. Here, the fragrance and the monomers are located in different compartments. This compartmentalization of the fragrance and the polymerization locus aims at preventing reaction of the fragrance molecules. This strategy allowed a high encapsulation efficiency of a fragrance, for which suspension polymerization was not possible, showing the protective role of the fact that polymerization takes place in a different compartment than the fragrance locus. The four systems arise from the choice of two monomers (Oligo(ethyleneglycol) methacrylate OEGMA and Methacrylamide MAM) and 
two cross-linkers (Tetra(ethyleneglycol) diacrylate TEGDA and N,N-Methylene-bisAcrylamide MbA). For all the four systems, the same double emulsion could be formulated. This shows the generality of the proposed strategy that constitutes a real advantage over the need of developing dedicated capsules for each considered fragrance. The initial double emulsion is composed of $75 \mathrm{wt} \%$ of globules in IPM, themselves composed of $20 \mathrm{wt} \%$ of fragrance droplets. Two stabilizers are required, Tergitol 15-s-12 in the aqueous phase to stabilize the fragrance droplets (around $1 \mu \mathrm{m}$ ) and PGPR in IPM to stabilize the large globules $(15 \mu \mathrm{m})$. As a consequence, a globule contains in average 675 droplets of fragrance. For each monomer/cross-linker couple, the maximum cross-linker concentration has been determined and is summarized in the following table (Table 3):

Table 3: Summary of the concentration possible for each system

\begin{tabular}{|c|c|c|}
\multicolumn{2}{c}{ OEGMA } & Methacrylamide MAM \\
\hline MbA & Up to $30 \mathrm{~mol}^{\%}$ & $5 \mathrm{~mol} \%$ \\
\hline TEGDA & Up to $10 \mathrm{~mol} \%$ & Up to $25 \mathrm{~mol}^{\%}$ \\
\hline
\end{tabular}

In all cases, the conversion rate was very satisfactory, and the obtained capsules exhibited interesting properties. They can be stored in closed vials keeping a high level of encapsulation efficiency and as required in many applications, they exhibit a slow and continuous release at room temperature (and a faster one at $40^{\circ} \mathrm{C}$ ) through diffusion in contact with air.

This kinetics of diffusive release can be modulated in a large range (an order of magnitude between the tightest and the most permeable capsules) by the choice of monomer and nature and/or concentration of cross-linker. The higher the total effective (physical and chemical) cross-linking, the slower the diffusion. Moreover, these capsules likely behave as small sponges as they release fragrance under mechanical stresses without capsules rupture. 
Consequently, the release can be both continuous and provoked on demand. The capsules exhibit therefore a triple possibility: they remain hermetic in storage conditions, they slowly release fragrance with a tunable kinetics and they exhibit a provoked boost effect. We think that these easily up scalable capsules exhibit a high application potential. Also, the choice of a fragrance, representative of real complex mixtures guarantees the applicability potential of the present work.

\section{Conflicts of interest}

The authors declare no conflict of interest

\section{Acknowledgements}

The authors would like to thank Takasago E.P.L and ANRT for financial support with contract number: 2014/1309. CNRS, Bordeaux University and IPB are also thanked for their financial contributions. The Cryo-SEM was performed at PLACAMAT with the help of Michel Martineau which is acknowledged by the authors. The authors would like to also thank Maud Bassereau (Takasago Europe Perfumery Laboratory) for her support for interpretation of some GC chromatograms

References

1. Green, B.K. and S. Lowell, Oil-containing microscopic capsules and method of making them, 1957, Patent Brevet US 2800457.

2. Doane, W.M., Encapsulation of pesticides in starch by extrusion. Industrial Crops and Products, 1992. 1(2-4): p. 83-87 DOI: 10.1016/0926-6690(92)90004-F.

3. Tsuiji, K., Microencapsulation of pesticides and their improved handling safety. Journal of Microencapsulation, 2001. 18(2): p. 137-147 DOI: 10.1080/026520401750063856.

4. Zhong, F., C. Yang, Q. Wu, S. Wang, L. Cheng, P. Dwivedi, Z. Zhu, T. Si, and R.X. $\mathrm{Xu}$, Preparation of pesticide-loaded microcapsules by liquid-driven coaxial flow focusing for controlled release. International Journal of Polymeric Materials and 
Polymeric Biomaterials, 2020. 69(13): p. 840-847 DOI: 10.1080/00914037.2019.1617710.

5. Zuidam, N.J. and V.A. Nedović, Encapsulation technologies for active food ingredients and food processing. Encapsulation Technologies for Active Food Ingredients and Food Processing. 2010. 1-400.

6. Singh, A., N. Dhiman, A.K. Kar, D. Singh, M.P. Purohit, D. Ghosh, and S. Patnaik, Advances in controlled release pesticide formulations: Prospects to safer integrated pest management and sustainable agriculture. Journal of Hazardous Materials, 2020. 385 DOI: 10.1016/j.jhazmat.2019.121525.

7. Nelson, G., Application of microencapsulation in textiles. International Journal of Pharmaceutics, 2002. 242(1-2): p. 55-62 DOI: 10.1016/S0378-5173(02)00141-2.

8. Yousefi, M., E. Khanniri, M. Shadnoush, N. Khorshidian, and A.M. Mortazavian, Development, characterization and in vitro antioxidant activity of chitosan-coated alginate microcapsules entrapping Viola odorata Linn. extract. International Journal of Biological Macromolecules, 2020. 163: p. 44-54 DOI: 10.1016/j.ijbiomac.2020.06.250.

9. Hameed, A., S.A. Hussain, S. Nosheen, Z. Muhammad, Y. Wu, S. Ullah, H.A.R. Suleria, and Y. Song, Microencapsulation of microbial antioxidants from Mucor circinelloides, their physico-chemical characterization, in vitro digestion and releasing behaviors in food. Applied Biological Chemistry, 2020. 63(1) DOI: 10.1186/s13765-020-00512-2.

10. Nollet, M., E. Laurichesse, S. Besse, O. Soubabère, and V. Schmitt, Determination of Formulation Conditions Allowing Double Emulsions Stabilized by PGPR and Sodium Caseinate to Be Used as Capsules. Langmuir, 2018. 34(8): p. 2823-2833 DOI: 10.1021/acs.langmuir.7b04085.

11. Dhakal, S.P. and J. He, Microencapsulation of vitamins in food applications to prevent losses in processing and storage: A review. Food Research International, 2020. 137 DOI: 10.1016/j.foodres.2020.109326.

12. Tözüm, M.S., C. Alkan, and S. Alay Aksoy, Preparation of poly(methyl methacrylateco-ethylene glycol dimethacrylate-co-glycidyl methacrylate) walled thermochromic microcapsules and their application to cotton fabrics. Journal of Applied Polymer Science, 2020. 137(24) DOI: 10.1002/app.48815.

13. Hong, K. and S. Park, Preparation of poly(L-lactide) microcapsules for fragrant fiber and their characteristics. Polymer, 2000. 41(12): p. 4567-4572 DOI: 10.1016/S00323861(99)00677-1.

14. Singh, M.N., K.S.Y. Hemant, M. Ram, and H.G. Shivakumar, Microencapsulation: A promising technique for controlled drug delivery. Research in Pharmaceutical Sciences, 2010. 5(2): p. 65-77.

15. Jin, Y., Q. Zhou, Z. Li, Z. Yang, and H.J.S. Fan, Calcium-cross linked polysaccharide microcapsules for controlled release and antimicrobial applications. Colloids and Surfaces A: Physicochemical and Engineering Aspects, 2020. 600 DOI: 10.1016/j.colsurfa.2020.125025.

16. Roucher, A., M. Morvan, D. Pekin, M. Depardieu, J.L. Blin, V. Schmitt, M. Konrad, J.C. Baret, and R. Backov, From Compartmentalization of Bacteria within Inorganic Macrocellular Beads to the Assembly of Microbial Consortia. Advanced Biosystems, 2018. 2(3) DOI: 10.1002/adbi.201700233.

17. Konuklu, Y., M. Ostry, H.O. Paksoy, and P. Charvat, Review on using microencapsulated phase change materials (PCM) in building applications. Energy and Buildings, 2015. 106: p. 134-155 DOI: 10.1016/j.enbuild.2015.07.019. 
18. Şahan, N. and H. Paksoy, Designing behenic acid microcapsules as novel phase change material for thermal energy storage applications at medium temperature. International Journal of Energy Research, 2020. 44(5): p. 3922-3933 DOI: 10.1002/er.5193.

19. Anaclet, P., M. Depardieu, E. Laurichesse, V. Julien, Y. Hung, V. Schmitt, and R. Backov, Bitumen@SiO 2 core-shell particles green synthesis towards flowable powdered bitumen and their binder applications. Colloids and Surfaces A: Physicochemical and Engineering Aspects, 2019. 570: p. 531-543 DOI: 10.1016/j.colsurfa.2019.03.048.

20. Anaclet, P., V. Julien, S. Mariotti, R. Backov, and V. Schmitt, Development of dispersible and flowable powdered bitumen. Journal of Cleaner Production, 2017. 141: p. 940-946 DOI: 10.1016/j.jclepro.2016.09.110.

21. Miles, J.M., B.M. Mitner, J. Brenner, and E.H. Polak, Encapsulated Perfumes in Aerosol Products. J. Soc.Cosmet Chem, 1971. 22: p. 655-666.

22. Velazquez, J.M., G. Marmolejo-Rivas, R.G. Riosdiaz, A.B. Alvarez, E.M. MarinCarrillo, and M.W. Glogowski, Encapsulated perfume particles and detergent compositions containing said particles 1999, Patent WO9955819 A1.

23. Hwang, J.S., J.N. Kim, Y.J. Wee, J.S. Yun, H.G. Jang, S.H. Kim, and H.W. Ryu, Preparation and characterization of melamine-formaldehyde resin microcapsules containing fragrant oil. Biotechnology and Bioprocess Engineering, 2006. 11(4): p. 332-336 DOI: 10.1007/BF03026249.

24. Hong, K. and S. Park, Melamine resin microcapsules containing fragrant oil: Synthesis and characterization. Materials Chemistry and Physics, 1999. 58(2): p. 128131 DOI: 10.1016/S0254-0584(98)00263-6.

25. Lee, H.Y., S.J. Lee, I.W. Cheong, and J.H. Kim, Microencapsulation of fragrant oil via in situ polymerization: effects of $\mathrm{pH}$ and melamine-formaldehyde molar ratio. Journal of Microencapsulation, 2002. 19(5): p. 559-569 DOI: 10.1080/02652040210140472.

26. Rochmadi, A. Prasetya, and W. Hasokowati, Mechanism of microencapsulation with Urea-Formaldehyde polymer. American Journal of Applied Sciences, 2010. 7(6): p. 739-745 DOI: 10.3844/ajassp.2010.739.745.

27. Jacquemond, M., N. Jeckelmann, L. Ouali, and O.P. Haefliger, Perfume-containing polyurea microcapsules with undetectable levels of free isocyanates. Journal of Applied Polymer Science, 2009. 114(5): p. 3074-3080 DOI: 10.1002/app.30857.

28. Warr, J., T. Ribaut, O. Anthony, and S. Fraser, Microcapsules, 2015, Patent EP2832442 (A1).

29. Warr, J., T. Ribaut, O. Anthony, and S. Fraser, Microcapsules, 2015, Patent EP2832441 (A1).

30. Warr, J., T. Ribaut, O. Anthony, and S. Fraser, Microcapsules, 2015, Patent EP2832440 (A1).

31. Whitaker, S. and M. Douglas, Stabilized perfume-containing microcapsules and method of preparing the same, 1991, Patent US5051305 (A).

32. Stasse, M., E. Laurichesse, T. Ribaut, O. Anthony, V. Héroguez, and V. Schmitt, Formulation of concentrated oil-in-water-in-oil double emulsions for fragrance encapsulation. Colloids and Surfaces A: Physicochemical and Engineering Aspects, 2020. 592: p. 124564.

33. Stasse, M., T. Ribaut, V. Schmitt, and V. Héroguez, Encapsulation of lipophilic fragrance by polymerization of the intermediate aqueous phase of an oil-in-water-inoil (O/W/O) double emulsion. Polymer Chemistry, 2019. 10: p. 4154-4162 DOI: 10.1039/C9PY00528E. 
34. Kuo, S.-W., H.-C. Kao, and F.-C. Chang, Thermal behavior and specific interaction in high glass transition temperature PMMA copolymer. Polymer, 2003. 44(22): p. 68736882 DOI: 10.1016/j.polymer.2003.08.026.

35. Laane, C., S. Boeren, K. Vos, and C. Veeger, Rules for optimization of biocatalysis in organic solvents. Biotechnology and Bioengineering, 1987. 30(1): p. 81-87 DOI: 10.1002/bit.260300112.

36. Mabille, C., F. Leal-Calderon, J. Bibette, and V. Schmitt, Monodisperse fragmentation in emulsions: Mechanisms and kinetics. Europhysics Letters (EPL), 2003. 61(5): p. 708-714 DOI: 10.1209/epl/i2003-00133-6.

37. George, J.D., C.J. Price, M.C. Marr, C.B. Myers, B.A. Schwetz, and J.J. Heindel, Evaluation of the development toxicity of methacrylamide and $N, N^{\prime}$ methylenebisacrylamide in Swiss Mice. Toxicological Sciences, 1998. 46(1): p. 124133 DOI: 10.1006/toxs.1998.2506.

38. Dintwa, E., E. Tijskens, and H. Ramon, On the accuracy of the Hertz model to describe the normal contact of soft elastic spheres. Granular Matter, 2008. 10(3): p. 209-221 DOI: 10.1007/s10035-007-0078-7.

39. León, G., N. Paret, P. Fankhauser, D. Grenno, P. Erni, L. Ouali, and D.L. Berthier, Formaldehyde-free melamine microcapsules as core/shell delivery systems for encapsulation of volatile active ingredients. RSC Adv., 2017. 7(31): p. 18962-18975 DOI: 10.1039/C7RA01413A.

40. Liao, Z., D. Xue, H. Li, and L. Shi, Fragrance-Containing Microcapsules Based on Interfacial Thiol-Ene Polymerization. Journal of Applied Polymer Science, 2016. 133(36) DOI: 10.1002/app.43905.

41. Chun, J.-Y., S.-K. You, M.-Y. Lee, M.-J. Choi, and S.-G. Min, Characterization of $\beta$ cyclodextrin Self-Aggregates for Eugenol Encapsulation. International Journal of Food Engineering, 2012. 8(2) DOI: 10.1515/1556-3758.2590.

42. Shao, Y., C. Wu, T. Wu, Y. Li, S. Chen, C. Yuan, and Y. Hu, Eugenol-chitosan nanoemulsions by ultrasound-mediated emulsification: Formulation, characterization and antimicrobial activity. Carbohydrate Polymers, 2018. 193: p. 144-152 DOI: 10.1016/j.carbpol.2018.03.101.

43. Shinde, U. and M. Nagarsenker, Microencapsulation of Eugenol by Gelatin-Sodium Alginate Complex Coacervation. Indian J Pharm Sci, 2011. 73(3): p. 311-315.

44. Bhandari, B.R., E.D. Dumoulin, H.M.J. Richard, I. Noleau, and A.M. Lebert, Flavor Encapsulation by Spray Drying: Application to Citral and Linalyl Acetate. Journal of Food Science, 1992. 57(1): p. 217-221 DOI: 10.1111/j.1365-2621.1992.tb05459.x.

45. Bertolini, A.C., A.C. Siani, and C.R.F. Grosso, Stability of Monoterpenes Encapsulated in Gum Arabic by Spray-Drying. Journal of Agricultural and Food Chemistry, 2001. 49(2): p. 780-785 DOI: 10.1021/jf000436y.

46. Bône, S., C. Vautrin, V. Barbesant, S. Truchon, I. Harrison, and C. Geffroy, Microencapsulated fragrances in melamine formaldehyde resins. CHIMIA International Journal for Chemistry, 2011. 65(3): p. 177-181.

47. Quellet, C., M. Schudel, and R. Ringgenberg, Flavors \& fragrance delivery systems. CHIMIA International Journal for Chemistry, 2001. 55(5): p. 421-428.

48. Fuchs, M., C. Turchiuli, M. Bohin, M.E. Cuvelier, C. Ordonnaud, M.N. PeyratMaillard, and E. Dumoulin, Encapsulation of oil in powder using spray drying and fluidised bed agglomeration. Journal of Food Engineering, 2006. 75(1): p. 27-35 DOI: 10.1016/j.jfoodeng.2005.03.047.

49. Pays, K., J. Giermanska-Kahn, B. Pouligny, J. Bibette, and F. Leal-Calderon, Coalescence in Surfactant-Stabilized Double Emulsions. Langmuir, 2001. 17(25): p. 7758-7769 DOI: 10.1021/la010735x. 
50. Pays, K., J. Giermanska-Kahn, B. Pouligny, J. Bibette, and F. Leal-Calderon, Double emulsions: how does release occur? Journal of controlled release, 2002. 79(1): p. 193205.

51. Nollet, M., M. Mercé, E. Laurichesse, A. Pezon, O. Soubabère, S. Besse, and V. Schmitt, Water fluxes and encapsulation efficiency in double emulsions: impact of emulsification and osmotic pressure unbalance. Soft Matter, 2016. 12(14): p. 34123424 DOI: 10.1039/C5SM03089G.

52. Chen, K., C. Xu, J. Zhou, R. Zhao, Q. Gao, and C. Wang, Multifunctional fabric coatings with slow-releasing fragrance and $U V$ resistant properties from ethyl cellulose/silica hybrid microcapsules. Carbohydrate Polymers, 2020. 232 DOI: 10.1016/j.carbpol.2019.115821.

53. Deng, R., Y. Wang, L. Yang, and C.D. Bain, In Situ Fabrication of Polymeric Microcapsules by Ink-Jet Printing of Emulsions. ACS Applied Materials and Interfaces, 2019. 11(43): p. 40652-40661 DOI: 10.1021/acsami.9b14417.

54. Ali, M., S.P. Meaney, M.J. Abedin, P. Holt, M. Majumder, and R.F. Tabor, Graphene oxide-silica hybrid capsules for sustained fragrance release. Journal of Colloid and Interface Science, 2019. 552: p. 528-539 DOI: 10.1016/j.jcis.2019.05.061.

55. Radulova, G.M., T.G. Slavova, P.A. Kralchevsky, E.S. Basheva, K.G. Marinova, and K.D. Danov, Encapsulation of oils and fragrances by core-in-shell structures from silica particles, polymers and surfactants: The brick-and-mortar concept. Colloids and Surfaces A: Physicochemical and Engineering Aspects, 2018. 559: p. 351-364 DOI: 10.1016/j.colsurfa.2018.09.079.

56. Lee, H., C.H. Choi, A. Abbaspourrad, C. Wesner, M. Caggioni, T. Zhu, and D.A. Weitz, Encapsulation and Enhanced Retention of Fragrance in Polymer Microcapsules. ACS Applied Materials and Interfaces, 2016. 8(6): p. 4007-4013 DOI: 10.1021/acsami.5b11351.

57. Wu, Y., J. Shen, V. Larcinese-Hafner, P. Erni, and L. Ouali, Hybrid microcapsules with tunable properties: Via Pickering emulsion templates for the encapsulation of bioactive volatiles. RSC Advances, 2016. 6(104): p. 102595-102602 DOI: 10.1039/c6ra21338c.

58. Ichiura, H., M. Takayama, N. Nishida, and Y. Otani, Interfacial polymerization preparation of functional paper coated with polyamide film containing volatile essential oil. Journal of Applied Polymer Science, 2012. 124(1): p. 242-247 DOI: 10.1002/app.33900.

59. Jonnalagadda, R.R., P. Velmurugan, G. Baskar, and B.U. Nair. Development of natural oil micro-emulsions for scent infused leathers. in 31st IULTCS Congress. 2011.

60. Destribats, M., V. Schmitt, and R. Backov, Thermostimulable wax@SiO2 core - Shell particles. Langmuir, 2010. 26(3): p. 1734-1742 DOI: 10.1021/la902828q.

61. Baillot, M., G. Hemery, O. Sandre, V. Schmitt, and R. Backov, Thermomagnetically Responsiver-Fe2O3@Wax@SiO2 Sub-Micrometer Capsules. Particle and Particle Systems Characterization, 2017. 34(10) DOI: 10.1002/ppsc.201700063.

62. Baillot, M., A. Bentaleb, E. Laurichesse, V. Schmitt, and R. Backov, Triggering the Mechanical Release of Mineralized Pickering Emulsion-Based Capsules. Langmuir, 2016. 32(16): p. 3880-3889 DOI: 10.1021/acs.langmuir.5b04687.

63. Jiang, H., L. Hong, Y. Li, and T. Ngai, All-Silica Submicrometer Colloidosomes for Cargo Protection and Tunable Release. Angewandte Chemie - International Edition, 2018. 57(36): p. 11662-11666 DOI: 10.1002/anie.201805968. 
64. Nollet, M., M. Depardieu, M. Destribats, R. Backov, and V. Schmitt, Thermoresponsive multi-cargo core shell particles. Particle and Particle Systems Characterization, 2013. 30(1): p. 62-66 DOI: 10.1002/ppsc.201200032.

65. Depardieu, M., M. Nollet, M. Destribats, V. Schmitt, and R. Backov, Thermostimulablewax@water@SiO2 multicore-shell capsules. Particle and Particle Systems Characterization, 2013. 30(2): p. 185-192 DOI: 10.1002/ppsc.201200069.

66. Shilpi, S., A. Jain, Y. Gupta, and S.K. Jain, Colloidosomes: An emerging vesicular system in drug delivery. Critical Reviews in Therapeutic Drug Carrier Systems, 2007. 24(4): p. 361-391 DOI: 10.1615/CritRevTherDrugCarrierSyst.v24.i4.20.

67. Dinsmore, A.D., M.F. Hsu, M.G. Nikolaides, M. Marquez, A.R. Bausch, and D.A. Weitz, Colloidosomes: Selectively permeable capsules composed of colloidal particles. Science, 2002. 298(5595): p. 1006-1009 DOI: 10.1126/science.1074868. 